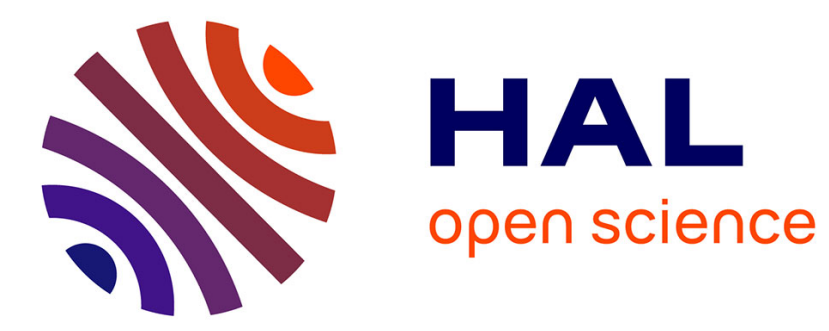

\title{
Thermodynamics of phase-separating nanoalloys: Single particles and particle assemblies
}

\author{
Mathieu Fèvre, Yann Le Bouar, Alphonse Finel
}

\section{To cite this version:}

Mathieu Fèvre, Yann Le Bouar, Alphonse Finel. Thermodynamics of phase-separating nanoalloys: Single particles and particle assemblies. Physical Review B: Condensed Matter and Materials Physics (1998-2015), 2018, 97, pp.195404-1 - 195404-13. 10.1103/PhysRevB.97.195404 . hal-01839959

\section{HAL Id: hal-01839959 https://hal.science/hal-01839959}

Submitted on 16 Jul 2018

HAL is a multi-disciplinary open access archive for the deposit and dissemination of scientific research documents, whether they are published or not. The documents may come from teaching and research institutions in France or abroad, or from public or private research centers.
L'archive ouverte pluridisciplinaire $\mathbf{H A L}$, est destinée au dépôt et à la diffusion de documents scientifiques de niveau recherche, publiés ou non, émanant des établissements d'enseignement et de recherche français ou étrangers, des laboratoires publics ou privés. 


\title{
Thermodynamics of phase-separating nanoalloys: Single particles and particle assemblies
}

\author{
Mathieu Fèvre, ${ }^{*}$ Yann Le Bouar, and Alphonse Finel \\ Laboratoire d'Étude des Microstructures, \\ CNRS, ONERA, Université Paris-Saclay, \\ BP72, 29, Avenue de la Division Leclerc, \\ 92322 Châtillon Cedex, France
}

(Dated: May 15, 2018) 


\begin{abstract}
The aim of this paper is to investigate the consequences of finite size effects on the thermodynamics of nanoparticle assemblies and isolated particles. We consider a binary phase separating alloy with a negligible atomic size mismatch and equilibrium states are computed using off-lattice Monte Carlo simulations in several thermodynamic ensembles. First, semi-grand canonical ensemble is used to describe infinite assemblies of particle with the same size. When decreasing the particle size, we obtain a significant decrease of the solid/liquid transition temperatures as well as a growing asymmetry of the solid state miscibility gap related to surface segregation effects. Second, a canonical ensemble is used to analyze the thermodynamic equilibrium of finite monodisperse particle assemblies. Using a general thermodynamic formulation, we show that a particle assembly may split into two sub-assemblies of identical particles. Moreover, if the overall average canonical concentration belongs to a discrete spectrum, the sub-assemblies concentrations are equal to the semi-grand canonical equilibrium ones. We also show that the equilibrium of a particle assembly with a prescribed size distribution combines a size effect and the fact that a given particle size assembly can adopt two configurations. Finally, we have considered the thermodynamics of an isolated particle to analyze whether a phase separation can be defined within a particle. When studying rather large nanoparticles, we found that the region in which a two-phase domain can be identified inside a particle is well below the bulk phase diagram but the concentration of the homogeneous core remains very close to the bulk solubility limit.
\end{abstract}

PACS numbers: 82.60.Qr, 64.75.Jk, 64.75.Gh, 64.60.De 


\section{INTRODUCTION}

Nanoalloys are metallic particles containing two or more elements with dimensions ranging typically from 1 to $20 \mathrm{~nm}$. These objects have properties, which are fundamentally different from those of discrete molecules and bulk systems. Nanoalloys have been the focus of intense academic research and have now many applications in different fields of science and technology such as in catalysis, magnetism and optics. ${ }^{1}$ The knowledge of the structural stability of nanoalloys ${ }^{2-4}$ is essential to guide the synthesis processes by the physical route and is also the first step towards the understanding of the aging of these nano-objects, an important question for the use of these objects in practical applications.

The first approach to model the stability of nanoalloys is based on a continuum description, only relevant for large enough particles. In these models, it is assumed that the structure of a nanoalloys can be decomposed in homogeneous regions (called phases) surrounded by surfaces or interfaces. The geometry of the homogeneous regions and the thermodynamical behavior of surfaces are assumed simple and characterized by a very limited number of parameters. Equilibrium is then defined as the minimum of the Gibbs energy when varying these parameters. Using these continuous models, the effect of the size and shape of the particles on the equilibrium structure has been demonstrated ${ }^{5-8}$ with, for example, the increase of solubility in nanoalloys and the decrease of melting temperatures. Size and shape dependent phase diagrams have been defined, in which equilibrium compositions after separation and solubility limits do not coincide. It has also been shown that the conservation of matter leads to constraints on the nucleation and growth of a new phase and to the existence of a critical particle size below which a two-phase state is unstable.

A more accurate description of the stability of nanoalloys is however provided by atomic approaches, using either molecular dynamics or Monte Carlo methods. These approaches can in principle explore all possible structures and configurations (core/shell, Janus-like, surface reconstructions, ...) and automatically include statistical fluctuations, which are required to analyze the stability of small systems, inasmuch the different thermodynamic ensembles of finite systems are not equivalent. These approaches have been used to analyze the stability of specific particles and to explain non-trivial transformations such as the

transition between the high miscibility in $\mathrm{Cs}_{3} \mathrm{Na}$ nanoparticles at low temperature and a demixtion at room temperature, ${ }^{9}$ the dynamical equilibrium of the outer shell of $\mathrm{Cu}-\mathrm{Ag}$ 
nanoalloys, ${ }^{10}$ the formation of core-shell in size mismatched nano-alloys,${ }^{11}$ and the ordering in Au-Pd nanoalloys. ${ }^{12}$

Using atomic approaches, the definition of a relevant size dependent phase diagram is much more complicated than with continuum approaches. First, in a finite size system, there is, strictly speaking, no phase transition because the partition function is analytic. Fortunately, even for nanometric particles, phase transitions can often be safely defined by rapid variations of thermodynamic quantities. ${ }^{13,14}$ Second, because statistical ensembles are not equivalent for finite size systems, the definition of an equilibrium state is only valid within a given thermodynamic ensemble. This also raises the question of the relevance of the computed equilibrium to analyze specific experimental results. Third, atomic models automatically incorporate the complexity of the thermodynamical behavior of interfaces, in particular segregation and wetting, during which a concentration excess appears at the surface. Due to the finite size of the system, the formation of such an excess necessarily implies a depletion in the core of the particle and therefore strongly impacts the stability of the particle.

Due to the high computational cost, atomic scale approaches have rarely been used to study the complete phase diagram of nanoalloys. One of the goals of the present work is to give a general picture of the phase diagram of nanoalloys through the computation of size dependent phase diagram spanning the whole composition and temperature ranges. To this end, we use the simple, flexible and low time consuming Lennard-Jones pair-wise interaction potential and off-lattice Monte Carlo calculations that can reproduce the stability of both solid and liquid phases and allow surface reconstructions. Such a pairwise potential is sufficient to address fundamental issues but is not appropriate to study specific metallic systems. This would require a more accurate description of the many body character of the chemical bonding, for example using tight binding or embedded-atom method (EAM) type potentials. Another aim of the present work is to present and compare several definitions of nanoalloys phase diagrams, based on several choices of thermodynamic ensembles. Finally, this work also presents the analysis of the stability of nanoparticle assemblies, including the case of a finite size distribution. The present paper does not consider the stability of small atomic clusters, for which the crystalline structure differs from the bulk one. ${ }^{13,15,16}$ This study focuses on the phase diagrams of binary alloys with a face centered cubic structure when the size mismatch between atoms is negligible (e.g., Rh-Pd, Ir-Pd). The methodology 
discussed in the following is however fully adapted to study the effects of elasticity on the solubility limits of nanolloys with an atomic size mismatch for which the strain have been shown to strongly impact the nanoparticle morphology. ${ }^{26}$

The paper is organized as follows. In Sec. II, we describe the interatomic potential and the procedures used to compute phase diagrams in the canonical and the semi-grand canonical ensembles. Sec. III is devoted to calculations performed in the semi-grand canonical ensemble, which is relevant for the stability of an infinite assemblies of particles with identical size. In Sec. IV, using the canonical ensemble, the stability of a finite assemblies of particles is discussed and the importance of the size distribution is demonstrated. Finally, the question of the phase separation within an isolated nanoparticle is addressed in Sec. V, before concluding remarks.

\section{TECHNICAL DETAILS AND METHODOLOGY}

\section{A. Thermodynamic ensembles}

Phase diagrams calculations have to be performed in specific thermodynamic ensembles. Here, we use the canonical and semi-grand canonical ensembles. In the canonical ensemble denoted here by $\left(\left\{N_{i}\right\}, P, T\right)$, the temperature $T$, the pressure $P$, the number $N_{i}$ of atomic sites of species $i$ in the M-component alloy are fixed quantities, where $\mathrm{M}$ is the number of chemical species. The mean concentration $\mathrm{c}_{i}$ of species $i$ is therefore a conserved quantity. In the semi-grand canonical ensemble, denoted here by $\left(N,\left\{\Delta \mu_{i}\right\}, P, T\right)$, the temperature $T$, the pressure $P$, the total number $N$ of atoms and the (M-1) alloy chemical potentials $\Delta \mu_{i}$ are

fixed quantities. $\Delta \mu_{i}=\mu_{i}-\mu_{\text {ref }}$ is the difference between the chemical potential $\mu_{i}$ of atomic species $i$ and the chemical potential of a reference one. Calculations in the $\left(\left\{N_{i}\right\}, P, T\right)$ and in the $\left(N,\left\{\Delta \mu_{i}\right\}, P, T\right)$ ensembles are realized with a Monte Carlo code which takes into account chemical relaxations and atomic displacements. In the $\left(\left\{N_{i}\right\}, P, T\right)$ ensemble, the Metropolis algorithm ${ }^{17}$ is applied to exchanges between particles to reproduce chemical correlations and to atomic displacements. This main loop is iterated until the Markov chain converges to equilibrium. Because atomic size effects are not considered in this work, the usual Markov steps on homogeneous strain have not been included. ${ }^{18}$ In the $\left(N,\left\{\Delta \mu_{i}\right\}, P, T\right)$ ensemble, we apply the same Metropolis algorithm, except that position exchanges between 
particles (swapping mechanism) are replaced by changes of atomic species on single sites (flipping mechanism). In the exchange mechanism, because we are interested in equilibrium but not kinetics, the choice of the two atoms is realized as follows : for each atom visited sequentially, a second atom is randomly chosen. The flipping mechanism is operated on a randomly chosen atom. Displacements are attempted sequentially on each atom of the simulation box. The displacement vector coordinates are randomly selected between -0.1 and 0.1A. As usual, we define a Monte Carlo time in terms of Monte Carlo Steps (MCS). In our calculations, one MCS corresponds to $10 \mathrm{~N}$ attempts of exchanges (or identity changes), and $10 N$ attempts of displacements, $N$ being the total number of atoms.

In this work, the calculations are performed at zero pressure. The reference case is a bulk alloy i.e. a large system with periodic boundary conditions where phase transitions and the phase diagram are clearly defined. Then, we analyze the equilibrium of finite spherical particles with diameters between $2 \mathrm{~nm}$ and $15 \mathrm{~nm}$. In the simulations, the initial configurations correspond to a cut of the face centered cubic lattice by a sphere with a core rich in $\mathrm{B}$ atoms and a shell rich in A atoms to reduce hysteresis effects and metastabilities (pinning, lattice friction). ${ }^{18}$

\section{B. Interatomic potential}

To study generic behaviors, we use a Lennard-Jones potential, which is very flexible in comparison with more realistic potentials in terms of the determination of the potential parameters and also very efficient from a computational point of view. The total energy of the system is given by the sum of pair interactions:

$$
V^{\alpha \beta}\left(r_{i j}\right)=-4 \varepsilon^{\alpha \beta}\left[\left(\frac{\sigma^{\alpha \beta}}{r_{i j}}\right)^{6}-\left(\frac{\sigma^{\alpha \beta}}{r_{i j}}\right)^{12}\right]
$$

where $r_{i j}$ is the distance between the atom $i$ (of type $\alpha$ ) and the $j$ atom (of type $\beta$ ). The parameter $\sigma^{\alpha \beta}$ controls the position of the energy minimum and the parameter $\epsilon^{\alpha \beta}$ its depth. For the pure $\alpha$-component, $\sigma^{\alpha \alpha}$ is related to the equilibrium lattice parameter and $\epsilon^{\alpha \alpha}$ to the cohesive energy. The melting temperature and the surface energy depend on both $\sigma^{\alpha \alpha}$ and $\epsilon^{\alpha \alpha}$. In order to avoid energy discontinuities at the cutoff distance $r_{c u t}$, the potentials are multiplied, for distances between $r_{c u t}-\Delta r$ and $r_{c u t}$, by the polynomial tapering function 

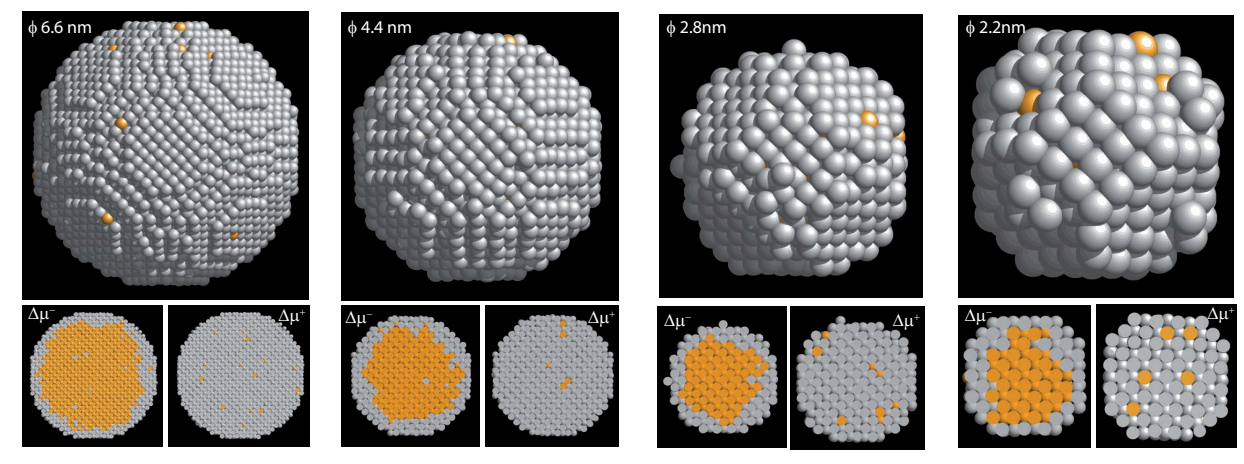

FIG. 1. Snapshots of four bimetallic particles of $6.6 \mathrm{~nm}, 4.4 \mathrm{~nm}, 2.8 \mathrm{~nm}$, and $2.2 \mathrm{~nm}$ in diameter from Monte Carlo simulations at $T=400 \mathrm{~K}$ in the semi-grand canonical ensemble. The light (dark) color corresponds to B (A) atoms respectively. The four particles contain 12137, 3565, 1058, and 429 atoms, respectively. Half sections of the particles are also presented for $\Delta \mu_{c}^{-}$and $\Delta \mu_{c}^{+}$(see text for detail).

$f(x)$ :

$$
f(x)=\left[8-15(2 x-1)+10(2 x-1)^{3}-3(2 x-1)^{5}\right] / 16
$$

where $x=\left[r-\left(r_{c u t}-\Delta r\right)\right] / \Delta r$. This procedure ensures that the potentials tend to zero smoothly over the distance $\Delta r$, which is typically chosen equal to $0.2 \AA$. The potentials are truncated between the 5th and 6th nearest neighbors of the face centered cubic (fcc) structure, which is the ground state for bulk alloys.

To decouple elastic effects and chemical effects, we choose to investigate in this paper an A-B alloy with no atomic size mismatch $(\Delta \mathrm{a} / \mathrm{a}=0) . \sigma^{A A}, \sigma^{A B}$ and $\sigma^{B B}$ are thus equal and adjusted to reproduce a particular lattice parameter $(0.36428 \mathrm{~nm})$. The values of $\epsilon^{\alpha \alpha}$ ( $\alpha=$ A or B) are chosen to reproduce two given melting temperatures close to $1300 \mathrm{~K}$ (1400K and 1155K). These values are representative of common face centered metals (e.g., $\mathrm{Cu}, \mathrm{Ag}, \mathrm{Ni}$, $\mathrm{Pt}$ ) but not specific to a particular one. $\epsilon^{A B}$ is chosen to ensure a phase separation at low temperature. Tab. I summarizes the parameter values as well as some energetic and elastic properties. The corresponding $(c, T)$ and $(\Delta \mu, T)$ phase diagrams for the bulk system are represented by black squares in Fig. 2 and Fig. 3. The miscibility gap is almost symmetric with respect to $c=0.5$. At this concentration, the transition temperature between the solid solution and the phase separation is $750 \mathrm{~K}$. More details can be found in Ref. 18. 


\begin{tabular}{lccc}
\hline \hline & $\mathrm{A}-\mathrm{A}$ & $\mathrm{A}-\mathrm{B}$ & $\mathrm{B}-\mathrm{B}$ \\
\hline$\varepsilon^{\alpha \beta}(\mathrm{eV})$ & 0.18 & 0.153 & 0.146 \\
$\sigma^{\alpha \beta}(\mathrm{nm})$ & 0.23508 & 0.23508 & 0.23508 \\
\hline$a(\mathrm{~nm})$ & 0.36428 & & 0.36428 \\
$\mathrm{E}_{c}(\mathrm{eV} / \mathrm{at})$. & -1.459 & & -1.186 \\
$\mathrm{Bulk} \mathrm{Mod.} \mathrm{(GPa)}$ & 155 & 126 \\
$\mathrm{C}_{11}(\mathrm{GPa})$ & 217 & 177 \\
$\mathrm{C}_{12}{ }^{\mathrm{a}}(\mathrm{GPa})$ & 124 & 100 \\
$\gamma_{111}($ J.m & & 1.2 \\
\hline \hline
\end{tabular}

${ }^{\text {a }}$ for pairwise potentials $\mathrm{C}_{44}=\mathrm{C}_{12}$ (Cauchy rule)

TABLE I. Lennard-Jones potential parameters (upper part) and corresponding quantities for the pure components (lower part) with interactions up to the fifth neighbors. The lattice parameters, the cohesive energies $\mathrm{E}_{c}$ and the elastic moduli calculated with a strict cutoff at zero temperature are given for the pure phases. $\gamma_{111}$ is the (111) surface energy at $0 \mathrm{~K}$.

\section{THERMODYNAMICS OF INFINITE PARTICLE ASSEMBLIES}

\section{A. Introduction}

In the semi-grand canonical ensemble, for large systems, phase transitions are associated with singularities (as function of $\Delta \mu_{i}, P$ or $T$ ) of quantities such as internal energy, concentrations or order parameters. When studying a demixtion process, the concentrations $c_{-}$and $c_{+}$corresponding to the limits of the miscibility gap are estimated by varying the alloy chemical potential $\Delta \mu$ to observe a finite jump of the concentration at a critical value $\Delta \mu_{c}$. In a previous study devoted to bulk alloys, we have developed a method based on the Gibbs-Thomson effect to minimize hysteresis and metastabilities taking place at low temperature (pinning, lattice friction). ${ }^{18}$ The method consists in analyzing the dynamics of a curved interface that separates a B-rich sphere embedded in an A-rich matrix and vice versa. The two critical values of the alloy chemical potential determined by finite increments on $\Delta \mu$ are then arithmetically averaged to obtain $\Delta \mu_{c}$. In the present study, because 
the analyzed systems are small, the characteristic length of the interface fluctuations is not negligible with respect to the particle size. As a consequence, the two initial configurations (A-rich and B-rich spheres) leads to the same behavior of the concentration variation as a function of the applied alloy chemical potential. Therefore, the limiting concentrations $c_{-}$ and $c_{+}$associated to the concentration jump at $\Delta \mu_{c}$, are simply identified as the concentrations corresponding to $\Delta \mu_{c}^{-}$and $\Delta \mu_{c}^{+}$, respectively, where $\Delta \mu_{c}^{-}\left(\Delta \mu_{c}^{+}\right)$is the alloy chemical potential just before (after) the concentration jump takes place.

For systems with free surfaces, the atomic sites close to the surface behave differently from the bulk ones due to their lower coordination numbers. Thus, apart from the bulk transition, the system may exhibit surface transitions, associated with successive critical

chemical potentials $\Delta \mu_{c} \cdot{ }^{10,19,20}$ Of course, the existence of a well defined sequence of surface transitions is likely to disappear at high temperature when spatial and chemical fluctuations of atomic sites are large enough.

When considering nano-objects, transitions are even more difficult to localize because they are smooth as a consequence of the analyticity of all statistical functions. ${ }^{24}$ However, when considering particles containing more than several hundreds of atoms, as it is the case in this study, transitions can usually still be clearly defined. Finally, another important point is that, for finite systems, the statistical ensembles are, strictly speaking, non-equivalent.

In this part, we first address the stability of a nano-particle in the semi-grand canonical ensemble. Then, the result is compared to the situation of a finite assemblies of particles with identical size in the canonical ensemble. Finally, the extension to an assemblies of particles differing in size is considered.

\section{B. Calculation in the semi-grand canonical ensemble}

We first consider a single particle in the semi-grand canonical ensemble. For a given particle size, the values of the critical chemical potential $\Delta \mu_{c}$ are searched in the $(\Delta \mu, T)$ parameter space. Fig. 1 shows snapshots of four particles of $6.6 \mathrm{~nm}, 4.4 \mathrm{~nm}, 2.8 \mathrm{~nm}$, and $2.2 \mathrm{~nm}$ in diameter at $T=400 \mathrm{~K}$. For each of these particle sizes, a run along increasing $\Delta \mu$ with small increments of $0.5 \mathrm{meV}$ reveals the occurrence of only one critical set $\left(\Delta \mu_{c}^{-}, \Delta \mu_{c}^{+}\right)$. Even for the smallest particle considered here (2.2 nm, 429 atoms) a chemical potential jump can be easily identified. The corresponding $(\Delta \mu, T)$ phase diagrams are shown in Fig. 2 . The 


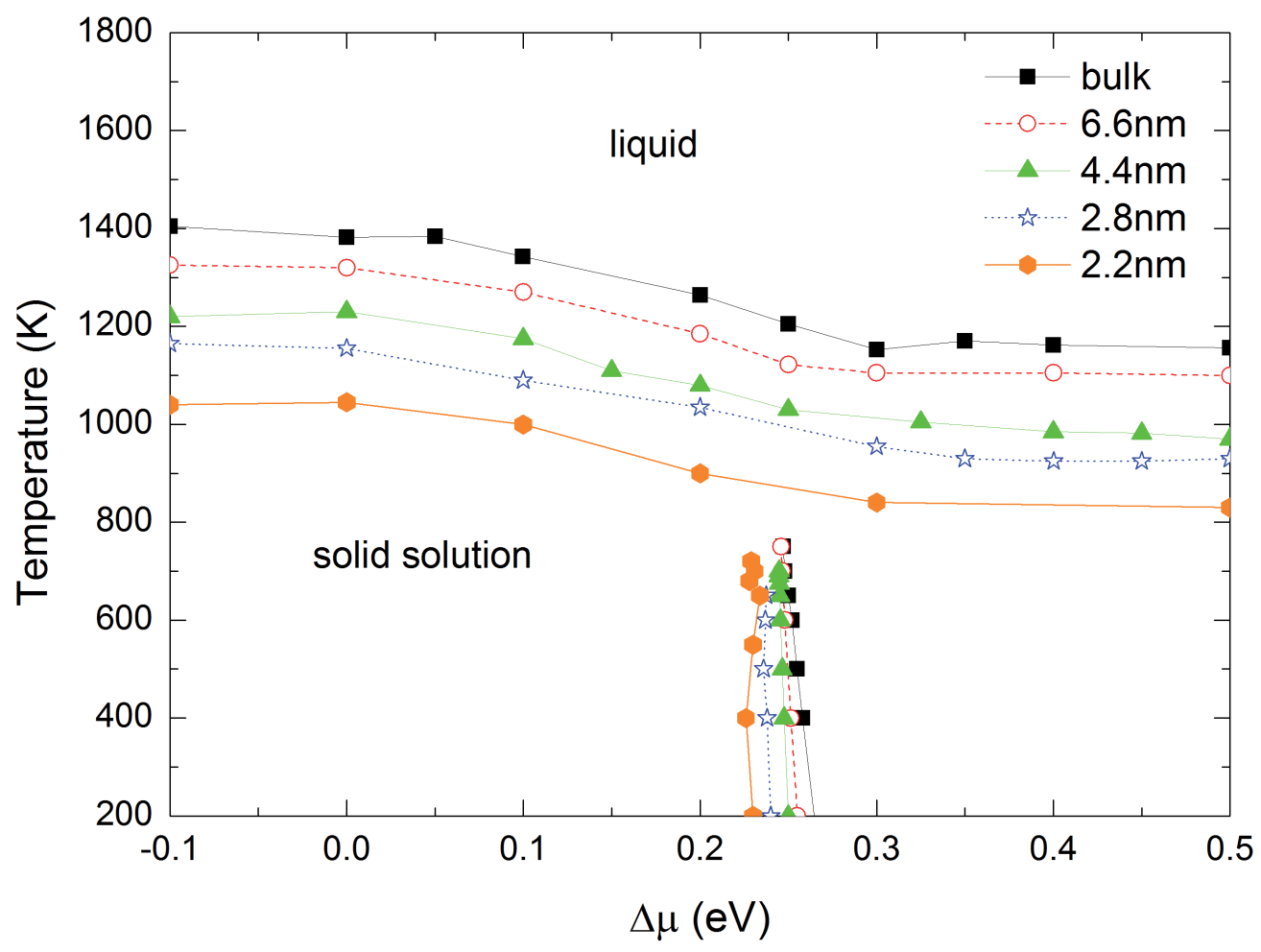

FIG. 2. Chemical potential-temperature phase diagrams for the bulk alloy and for spherical nanoparticles with diameters equal to $6.6,4.4,2.8$, and $2.2 \mathrm{~nm}$.

transition temperature between the liquid and the solid decreases with the particle size. The solid-solid transitions are also affected but only slightly: the critical value of the chemical potential of $2.2 \mathrm{~nm}$ particles is $10 \%$ lower than the bulk alloy one and the temperature corresponding to the top of the miscibility gap is lowered by only $3 \%$. However, in the $(c, T)$ phase diagrams, represented in Fig. 3, the miscibility gap is much more affected by size effects : it becomes asymmetric with respect to the equiatomic concentration and shrinks when the particle size decreases. This effect is related to the difference $\Delta \gamma$ between the surface energies of A and B atoms, which amounts to $200 \mathrm{~mJ}^{-\mathrm{m}^{-2}}$ for a (111) surface at $0 \mathrm{~K}$ (see Tab. I). We indeed checked that the miscibility gap remains symmetric if $\Delta \gamma$ is set to zero. This condition is easily imposed within the Lennard-Jones approach, as it amounts to simply impose $\epsilon^{A A}=\epsilon^{B B}$. We have also noted that the asymmetry of the miscibility gap is not linked to surface segregation effects, as, when $\Delta \gamma=0$, segregation of the minority species 


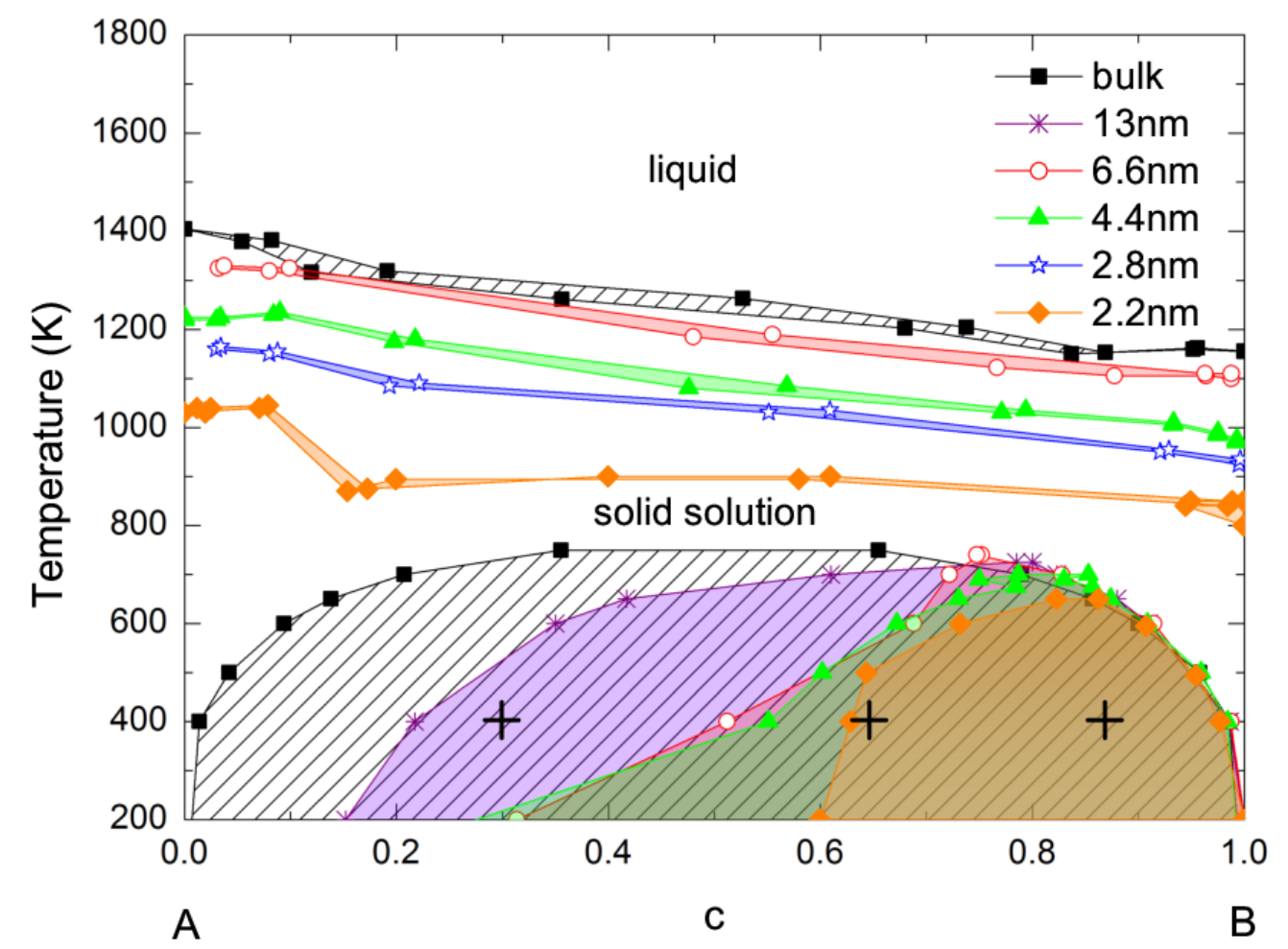

FIG. 3. Composition-temperature phase diagrams computed within the semi-grand canonical ensemble for the bulk alloy and for spherical nanoparticles with diameters equal to 13, 6.6, 4.4, 2.8, and $2.2 \mathrm{~nm}$. Shaded areas correspond to two-phase domains.

does exist and is still observed.

The knowledge of these size-dependent phase diagrams may be a useful tool to interpret the observed configurational states of large monodisperse assemblies of nanoparticles, if of course the synthesis conditions are such that the assembly reaches its equilibrium state. The semi-grand canonical thermodynamics of a single particle is formally equivalent to the canonical thermodynamics of an infinite assembly of particles with the same size, which only interact through the exchange of atoms. Therefore, in the single-phase domains of the phase diagram presented in Fig. 3, infinite assemblies feature only one type of particles, whereas in the two-phase domains, assemblies exhibit two populations. In the latter case, the equilibrium concentrations of each particle type are given by the limits of the miscibility gap and the fraction of particles of each type is given by the lever rule. 
Inside a given particle, the concentration fields are of course inhomogeneous and depend on temperature. In our calculations, we consider spherical particles and we observe that the concentration fields have a radial symmetry at equilibrium. As shown in Fig. 4, at $400 \mathrm{~K}$, the concentration profile associated with $\Delta \mu_{c}^{-}$exhibits a $4 \mathrm{~nm}$ large homogeneous zone poor in B atoms around the center of the particle (core), followed by a $0.5 \mathrm{~nm}$ thick interface and terminates by a surface rich in B (shell). For $\Delta \mu_{c}^{+}$, the concentration profile is quasi-homogeneous through the particle. At $750 \mathrm{~K}$, the concentration profiles exhibit a $1 \sim 2 \mathrm{~nm}$ large homogeneous zone close to the center of the particle and then a diffuse transition up to the surface, which is rich in B. These observations indicate that, within the miscibility gap, the configurational states of the particles at low temperature (coreshell like) are qualitatively very different from those observed at high temperature. In Fig. 5, the concentrations measured close the center of the particles (in the homogeneous zones) for $\Delta \mu_{c}^{-}$and $\Delta \mu_{c}^{+}$are superimposed to the bulk alloy $(c, T)$ phase diagram for solidsolid transformations. We observe that they are close to the limits of the bulk miscibilty gap. In other words, within the miscibility gap associated to nanoparticles, the equilibrium concentrations at the center of the particles differ only slightly from their bulk equilibrium values. For example, for assemblies of $6.6 \mathrm{~nm}$ particles, concentrations at the center only very slightly deviate from those of the bulk alloy (Fig. 5), even though the corresponding phase diagram is clearly very different from the bulk one (Fig. 3).

The previous calculations and conclusions pertain to a situation where a large number (possibly infinite) of monodisperse particles reach collectively a thermodynamical equilibrium. In the next section, we investigate the collective behavior of a small number of particles. This of course requires the use of the canonical ensemble.

\section{CANONICAL ENSEMBLE : FINITE ASSEMBLIES OF PARTICLES}

\section{A. Finite assemblies of particles with identical size}

We consider a finite assembly of particles in interaction in the canonical ensemble, where the number of $\mathrm{A}$ and $\mathrm{B}$ atoms in the assembly, the temperature and the pressure are fixed quantities. In order to make a link between the equilibrium of a single particle in the semigrand canonical ensemble and the equilibrium of the particles in the canonical ensemble, we 


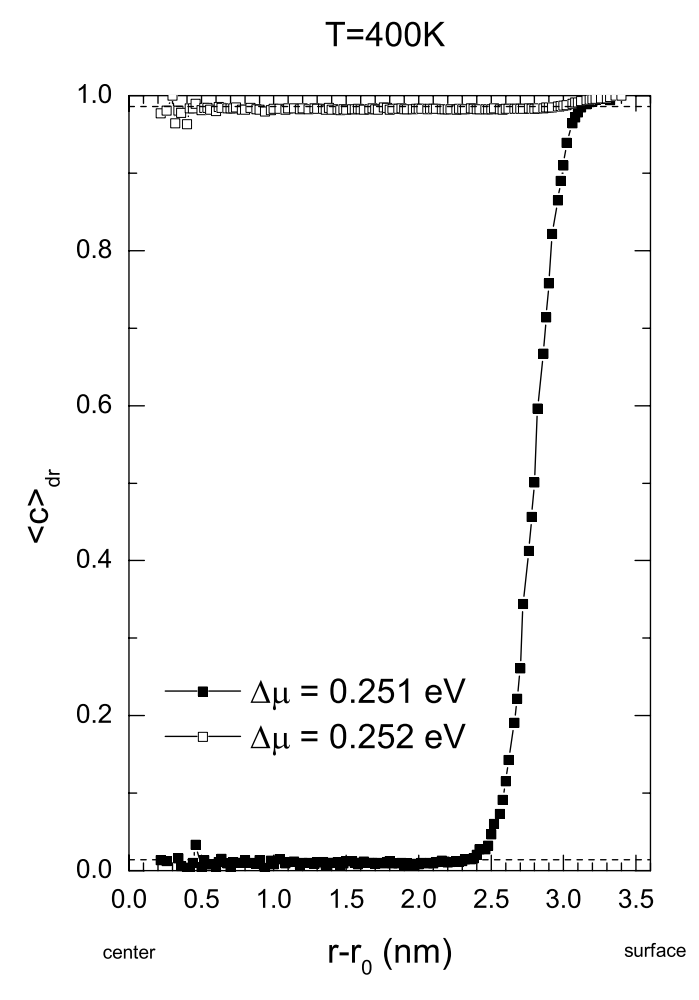

(a)

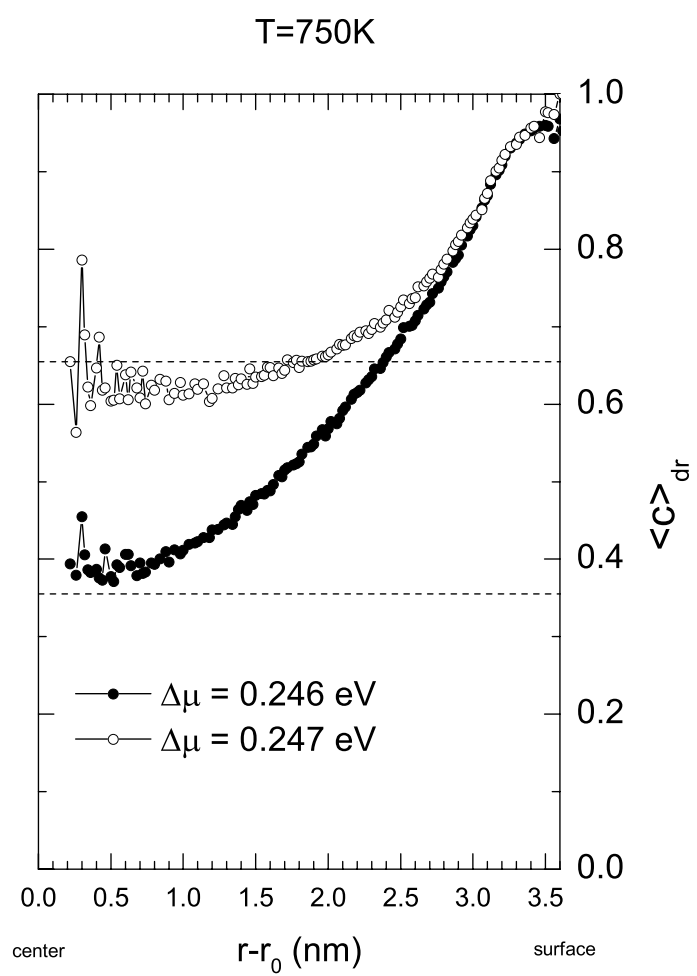

(b)

FIG. 4. Averaged radial concentration profiles of a $6.6 \mathrm{~nm}$ particle for $\Delta \mu_{c}^{-}$(open symbols) and $\Delta \mu_{c}^{+}$(close symbols). Figure 4(a) corresponds to calculations at 400K and Fig. 4(b) to calculations at $750 \mathrm{~K}$. Dashed lines correspond to the bulk alloy equilibrium concentrations.

investigate a situation where all the particles have the same size, the same shape and the same number of atomic sites.

In the semi-grand canonical ensemble, the relevant thermodynamical function is the grand potential $G(\Delta \mu)$, which is related to the canonical free energy $F(c)$ of a single particle by:

$$
G(\Delta \mu)=\min _{c}\{F(c)-\Delta \mu c\}
$$

We are interested here in a situation where the grand canonical equilibrium state is degenerated. In other words, the chemical potential $\Delta \mu$ is equal to its critical value $\Delta \mu_{c}$ and the concentration of the two degenerated states are such that : 


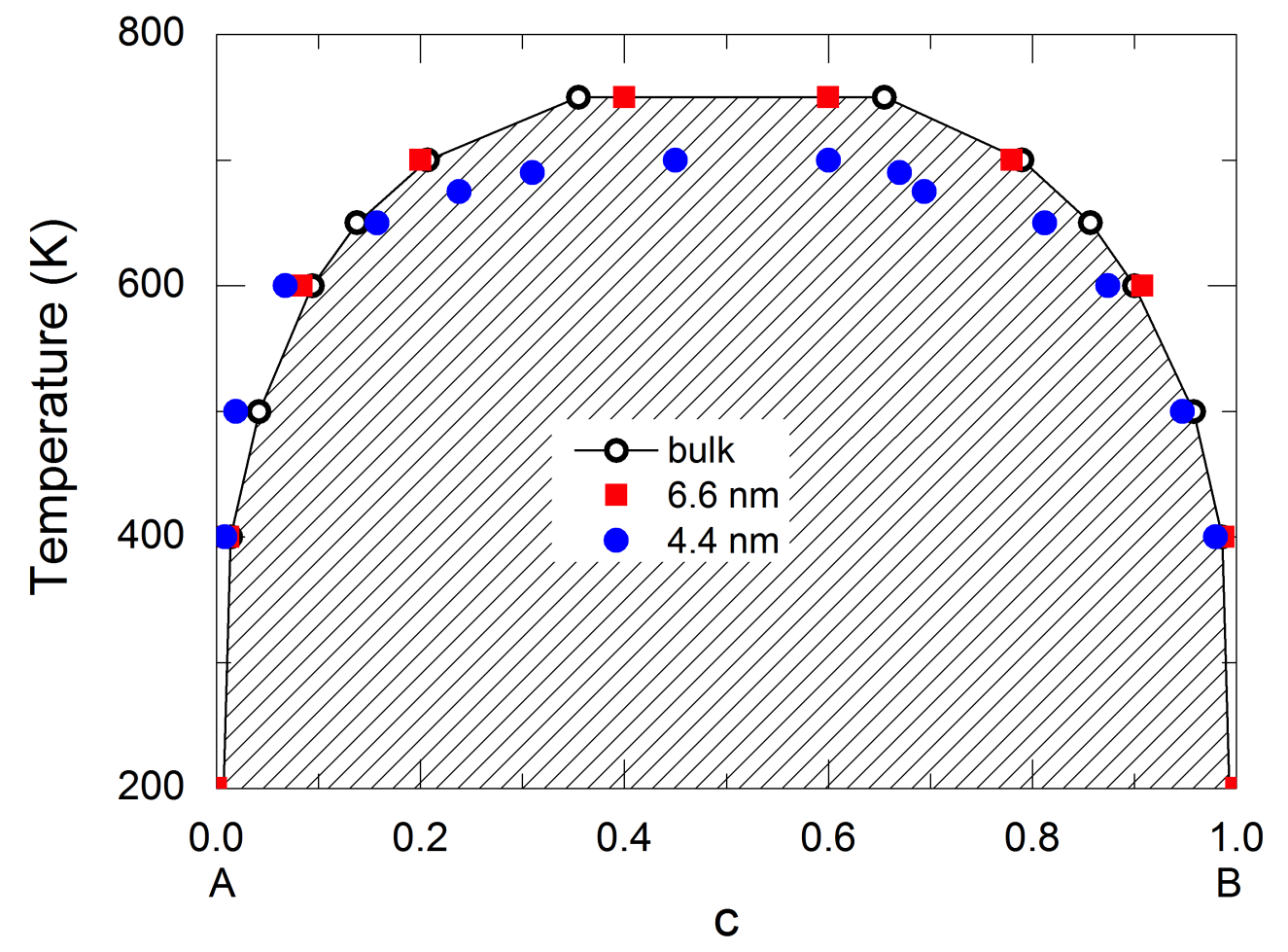

FIG. 5. Concentrations calculated at the center of particles with diameters equal to $6.6 \mathrm{~nm}$ and $4.4 \mathrm{~nm}$ (close symbols) superimposed on the corresponding bulk alloy $(c, T)$ phase diagram.

$$
F\left(c_{1}\right)-\Delta \mu_{c} c_{1}=F\left(c_{2}\right)-\Delta \mu_{c} c_{2}
$$

which, together with Eq. 3 leads to :

$$
\left.\frac{\partial F}{\partial c}\right|_{c_{1}}=\left.\frac{\partial F}{\partial c}\right|_{c_{2}}=\frac{F\left(c_{1}\right)-F\left(c_{2}\right)}{c_{1}-c_{2}}=\Delta \mu_{c}
$$

We consider now a finite monodisperse assembly of $N$ particles in the canonical ensemble. The total free energy of this assembly is given by:

$$
F_{\text {tot }}\left(\left\{c_{i}\right\}\right)=\sum_{i=1}^{N} F\left(c_{i}\right)
$$




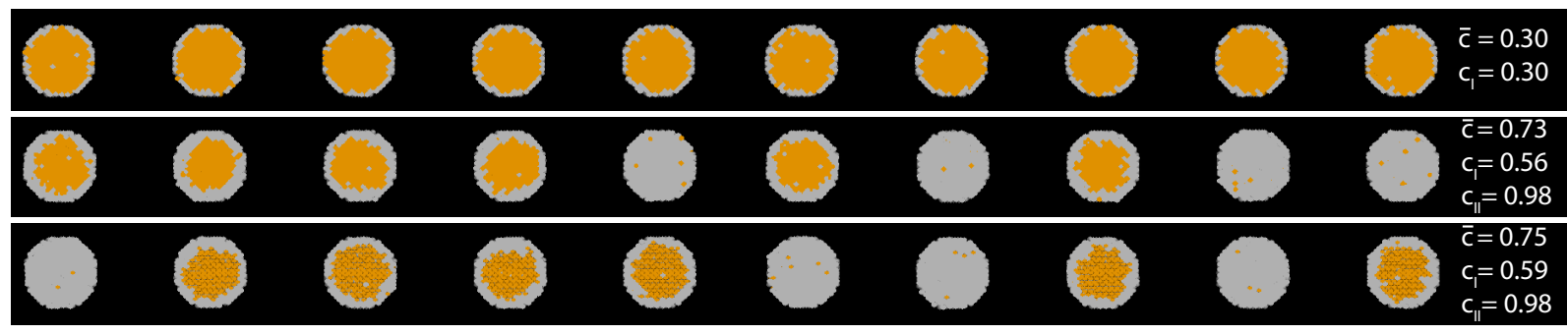

FIG. 6. Half sections of an assembly of ten particles obtained by Monte Carlo simulations at 400K in the canonical ensemble for three imposed total concentrations : $c_{t o t}=0.30,0.73$ and 0.75 from top to bottom. The light (dark) color corresponds to B (A) atoms respectively. The concentration of each particle population is also specified.

where $c_{i}$ is the average concentration of particle $i$. The additive property of $F_{t o t}$ is linked to two assumptions. First, there are no direct chemical interactions between atoms of different particles through the interatomic potential. The second requirement is less straightforward. The identification of an average concentration $c_{i}$ for a given particle and its associated canonical free energy $F\left(c_{i}\right)$ requires that the average concentration can be defined along a sufficiently long time sequence and that its fluctuations are small enough. This obviously requires that the particles are large enough, but also that the number of particles is sufficiently high in order to make the concentration fluctuations between particles nearly independent.

We now address the following question : in which circumstances does the canonical assembly of $N$ particles split in two sub-assemblies of $N_{1}$ and $N_{2}$ particles (with $N_{1}+N_{2}$ $=N$ ), characterized by two different concentrations $c_{1}$ and $c_{2}$, that are associated with the degenerated grand canonical equilibrium ?

The answer to this simple problem proceeds as follows. Under the previous assumptions, the equilibrium state $\left\{c_{i}^{e q}\right\}$ of our canonical ensemble is given by applying the variational principle on the free energy defined in Eq. 6 :

$$
F_{\text {tot }}\left(\left\{c_{i}^{e q}\right\}\right)=\min _{c_{i}} \sum_{i=1}^{N} F\left(c_{i}\right)
$$

under the constraint: 


$$
\sum_{i=1}^{N} c_{i}=N \bar{c}
$$

where $\bar{c}$ is the overall average concentration. This problem is equivalent to minimizing the functional $\mathcal{G}\left(\left\{c_{i}\right\}, \lambda\right)$, where $\lambda$ is a Lagrange parameter :

$$
\mathcal{G}\left(\left\{c_{i}\right\}, \lambda\right)=F\left(c_{i}\right)-\lambda\left(\sum_{i=1}^{N} c_{i}-N \bar{c}\right)
$$

The equilibrium concentrations should therefore verify :

$$
\left.\frac{\partial F}{\partial c}\right|_{c_{i}^{e q}}=\lambda
$$

In the present situation (a binary alloy that displays a miscibility gap), the generic shape of the low temperature free energy curve displays only two wells. Therefore, for a given (Lagrange parameter) $\lambda$, their exist only two different concentrations $c_{I}$ and $c_{I I}$ such that :

$$
\left.\frac{\partial F}{\partial c}\right|_{c_{I}}=\left.\frac{\partial F}{\partial c}\right|_{c_{I I}}=\lambda
$$

Each particle will now adopt one of these two concentrations. If $N_{I}$ (resp. $N_{I I}$ ) is the number of particles with concentration $c_{I}$ (resp. $c_{I I}$ ), the quantity $\mathcal{G}$ introduced above becomes a function of $N_{I}$ and $N_{I I}$ :

$$
\begin{aligned}
\mathcal{G}( & \left.N_{I}, N_{I I}, \lambda\right)=N_{I} F\left(c_{I}\right)+ \\
& N_{I I} F\left(c_{I I}\right)-\lambda\left(N_{I} c_{I}+N_{I I} c_{I I}-N \bar{c}\right)
\end{aligned}
$$

The next step of our variational procedure consists in minimizing this function with respect to the integers $N_{I}$ and $N_{I I}$. As their sum is constant, when they reach the values that minimize $\mathcal{G}\left(N_{I}, N_{I I}, \lambda\right), N_{I}$ and $N_{I I}$ must verify the following equations :

$$
\begin{aligned}
& \mathcal{G}\left(N_{I}, N_{I I}, \lambda\right) \leq \mathcal{G}\left(N_{I}-1, N_{I I}+1, \lambda\right) \\
& \mathcal{G}\left(N_{I}, N_{I I}, \lambda\right) \leq \mathcal{G}\left(N_{I}+1, N_{I I}-1, \lambda\right)
\end{aligned}
$$

This translates to :

$$
\begin{aligned}
F\left(c_{I}\right)-\lambda c_{I} & \leq F\left(c_{I I}\right)-\lambda c_{I I} \\
F\left(c_{I I}\right)-\lambda c_{I I} & \leq F\left(c_{I}\right)-\lambda c_{I}
\end{aligned}
$$


which implies :

$$
F\left(c_{I}\right)-\lambda c_{I}=F\left(c_{I I}\right)-\lambda c_{I I}
$$

The canonical concentrations $c_{I}$ and $c_{I I}$ should therefore verify simultaneously Eq. 11 and Eq. 17 :

$$
\left.\frac{\partial F}{\partial c}\right|_{c_{I}}=\left.\frac{\partial F}{\partial c}\right|_{c_{I I}}=\frac{F\left(c_{I}\right)-F\left(c_{I I}\right)}{c_{I}-c_{I I}}
$$

This equation is identical to Eq. 5. Therefore, if a solution to our initial problem exists, the concentrations $c_{I}$ and $c_{I I}$ of the two sub-assemblies of our canonical ensemble will be necessarily equal to the concentrations $c_{1}$ and $c_{2}$ that are associated to the degenerated grand canonical equilibrium:

$$
\begin{gathered}
c_{I}=c_{1} \\
c_{I I}=c_{2}
\end{gathered}
$$

Of course, as the concentration is a conserved quantity, a necessary condition for this solution to exist is that the solutions $N_{I}$ and $N_{I I}$ of the linear system:

$$
\left\{\begin{array}{c}
c_{1} N_{I}+c_{2} N_{I I}=N \bar{c} \\
N_{I}+N_{I I}=N
\end{array}\right.
$$

are integers. This requires that the average concentration $\bar{c}$ can be written as :

$$
\bar{c}=c_{1}+\frac{c_{2}-c_{1}}{N} k \quad ; \quad k=0,1, \cdots, N
$$

In summary, if the concentration $\bar{c}$ belongs to the discrete spectrum defined by Eq. 19, the canonical particle assembly of $N$ particles will split, at the equilibrium, into two subsets associated with the degenerated equilibrium concentrations $c_{1}$ and $c_{2}$ identified in the semi-grand canonical ensemble and the fraction of particles of each type will be given by the lever rule.

In order to confirm this analysis, we now proceed to a numerical study of the thermodynamical behavior of a finite set of particles. Two different situations are considered: first, a set of ten particles with a diameter of $4.4 \mathrm{~nm}$ and second, an assembly of 4 particles with a diameter equal to $2.2 \mathrm{~nm}$. 
The Monte Carlo results obtained for the set of ten identical $4.4 \mathrm{~nm}$ particles are represented in Fig. 6. Three average concentrations $(\bar{c}=0.30,0.728$ and 0.749$)$ have been investigated at the fixed temperature $\mathrm{T}=400 \mathrm{~K}$. When the average concentration $\bar{c}$ sits in the single-phase domain identified within the semi-grand canonical ensemble $(\bar{c}=0.30)$, we naturally observe a single particle state. Conversely, when $\bar{c}$ lies within the two-phase domain $\left(c_{1} \leq \bar{c} \leq c_{2}\right.$ with $c_{1}=0.56$ and $\left.c_{2}=0.98\right)$, the assembly splits into two subsets of particles, with specific concentrations $c_{I}$ and $c_{I I}$ (see Fig. 6), in agreement with the previous analysis. Moreover, when $\bar{c}$ fulfills the quantification requirement given by Eq. $19(\bar{c}=0.728)$, the observed canonical concentrations $c_{I}$ and $c_{I I}$ correspond exactly to the semi-grand canonical ones, $c_{1}$ and $c_{2}$, as predicted above. In contrast, when $\bar{c}$ deviates from the discrete spectrum $(\bar{c}=0.749)$, the equilibrium concentrations differ definitively from the semi-grand canonical ones, as expected. We now analyze the set of four identical particles with a diameter equal to $2.2 \mathrm{~nm}$ and for $\mathrm{T}=400 \mathrm{~K}$. The aim is to enhance the quantification effects associated with Eq. 19, and also, to investigate numerically the influence of a smaller particle size. According to the results presented in Fig. 3, the limits of the two-phase domain calculated in the semi-grand canonical ensemble for a particle size of $2.2 \mathrm{~nm}$ are $c_{1}=0.63$ and $c_{2}=0.98$. We have investigated many average concentrations $\bar{c}$ in between 0.5 to 1 and it appears that the 4-particle assembly may adopt qualitatively three different behaviors which are now described using the three selected concentrations $\bar{c}=0.805,0.7875$ and 0.77 . The overall behavior will be discussed at the end of this section.

Fig. 7 represents for each of the three concentrations $\bar{c}$ given above, the time evolution of the four particle concentrations observed in the canonical ensemble as well as the corresponding concentration distribution. At any time step, the assembly splits into two subsets with two concentrations $c_{I}$ and $c_{I I}$, as anticipated before. Due to the surface energy difference between $\mathrm{A}$ and $\mathrm{B}$ atoms, $c_{I I}$ is always close to $c_{2}$ (see section III B). In contrast, $c_{I}$ may strongly deviate from $c_{1}$. When $\bar{c}=0.805$, the requirement of Eq. 19 is fulfilled with $N_{I}=N_{I I}=2$ and we observe (Fig. 7a) that the canonical concentrations $c_{I}$ and $c_{I I}$ are equal to the semi-grand canonical concentrations $c_{1}$ and $c_{2}$ respectively, in agreement with the analysis presented above. The next situation, $\bar{c}=0.7875$, corresponds to a case where $\bar{c}$ deviates slightly from the quantification case just described. Consequently, we expect that the assembly still splits into two subsets with $N_{I}=N_{I I}=2$ but with canonical concentrations $c_{I}$ and $c_{I I}$ that differ from the semi-grand canonical concentrations $c_{1}$ and $c_{2}$. Indeed, 

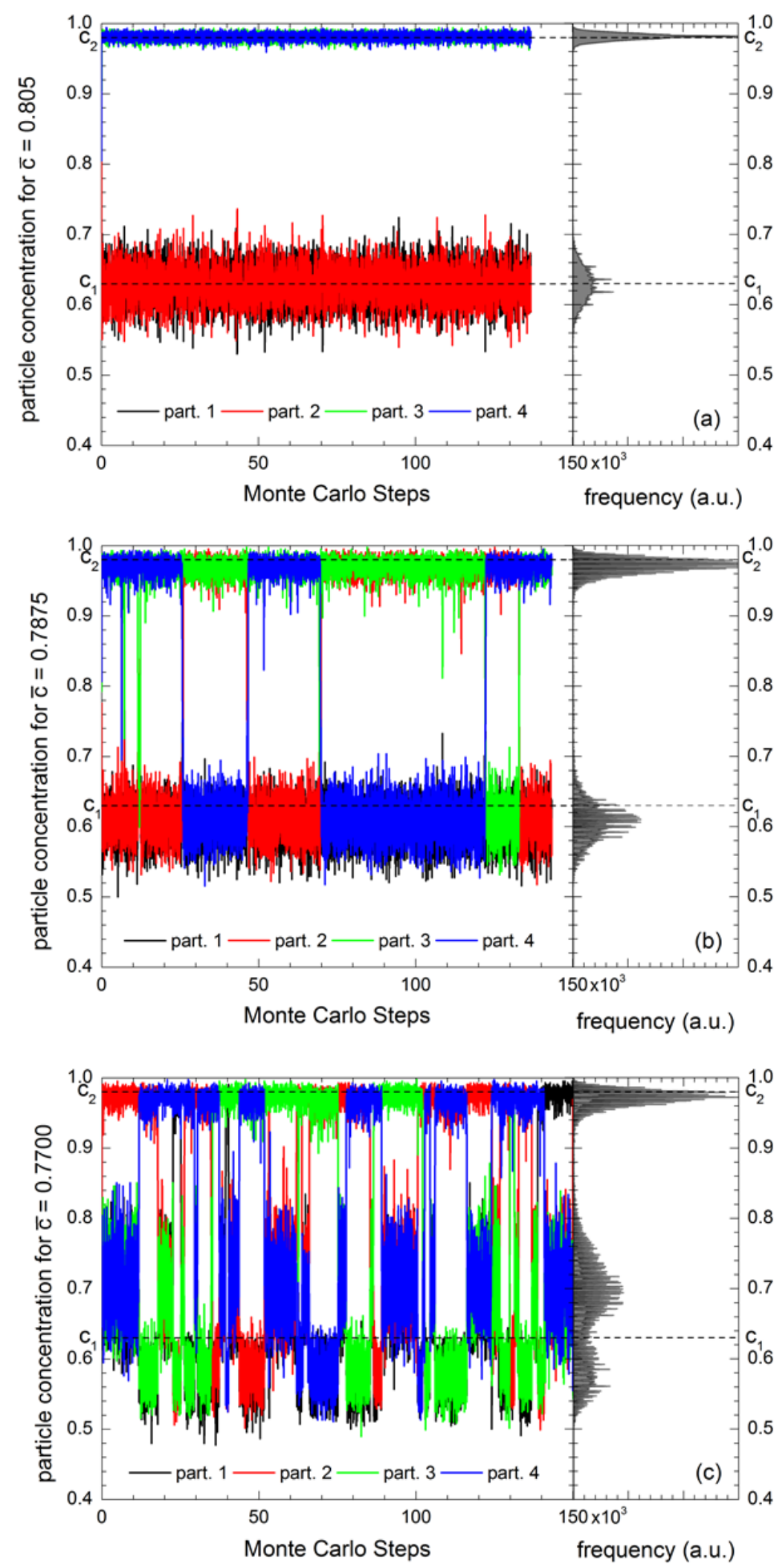

FIG. 7. Time evolution (in Monte Carlo Steps) and partial distribution of the particle concentrations in an assembly of four particles with diameters equal to $2.2 \mathrm{~nm}$, at $400 \mathrm{~K}$, for three average concentrations ( $\bar{c}=0.805,0.7875,0.7700$ from top to bottom). 


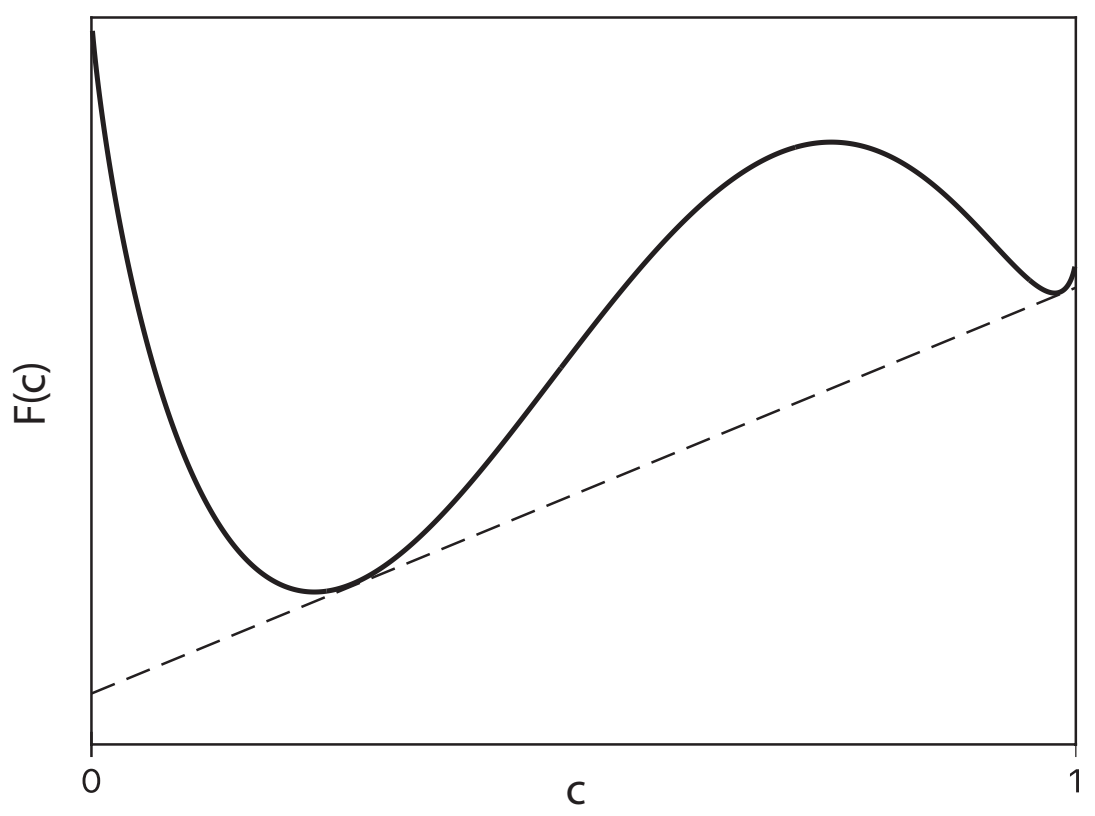

FIG. 8. Generic free energy curve of a single particle (see text for detail).

in Fig. 7b, we observe that $c_{I}=0.61$ and $c_{I I}=0.97$. The behavior is generic to a situation which is close but not equal to a quantification case. We also note that each of the four particle does not stay in the same state but instead switches between the two concentration $c_{I}$ and $c_{I I}$, but this switching event preserves the $N_{I}=N_{I I}=2$ partition. The last situation, $\bar{c}=0.77$, corresponds to a case which is in between two quantification values. More precisely, the next lower quantified concentration is $\bar{c}=0.7175$ for which $N_{I}=3$ and $N_{I I}=1$ and the next higher quantified concentration is $\bar{c}=0.805$ for which $N_{I}=2$ and $N_{I I}=2$. As consequence, for the considered concentration $\bar{c}=0.77$, the 4-particle assembly switches as a function of time between those two distributions as seen in Fig. 7c.

We also clearly observe in Fig. 7 that, within a given distribution $\left(N_{I}, N_{I I}\right)$, the concentration of each particle fluctuates around $c_{I}$ or $c_{I I}$. These fluctuations reach $\pm 10 \%$ around $c_{I}$ for the $2.2 \mathrm{~nm}$ particle considered in Fig. $7 \mathrm{c}$ and decrease as the particle size increases (not shown). These fluctuations and their amplitudes may be explained through a qualitative analysis of the canonical free energy $F(c)$ as a function of its average concentration $c$ (see Eq. 6 and the corresponding discussion). In the present situation where the thermodynamical properties of a particle is governed by the presence of a miscibility gap, the free energy $F(c)$ displays generically a two-well shape qualitatively displayed in Fig. 8. When a given par- 
ticle is thermodynamically linked to others within a canonical ensemble, its concentration, which is not fixed, can fluctuate around an average value. These fluctuations are controlled by the second derivative of $F(c)$. When the average concentration is close to 1 we clearly anticipate that the second derivatives are large and therefore the fluctuations should be small. This is the reason why, in all the cases shown in Fig. 7, the fluctuations around the average concentrations $c_{I I}$ (which are close to $c_{2}=0.98$ ) are small. The fluctuations around the other average concentration displayed by the particle (i.e. $c_{I}$ ) are much stronger because the second derivative of the free energy $F(c)$ is smaller (see Fig. 8). We also note that the fluctuations around $c_{I}$ increase with $c_{I}$ and that the fluctuations around $c_{I I}$ increase when $c_{I I}$ decreases. The reason is that, due to its double well shape and provided we consider a concentration domain around its minimum, the second derivative of the free energy $F(c)$ generically decreases when the average concentration $c$ comes closer to the middle of the two-phase grand canonical domain (see Fig. 8).

On the right hand side of Fig. 7, we observe that, on top of their overall shape, the concentration distributions display several extremely sharp peaks at specific positions. This shows that some particle configurations are observed with higher probabilities. We have verified that the position of these concentration peaks are not influenced by the number of particle in the assembly nor by temperature but by their size. Therefore we conclude that this specific configurations correspond to local energy minima. This illustrates the importance of the geometric structure when considering small nanoparticles, a feature extensively discussed in the literature. ${ }^{21,22}$

We now present the overall 4-particle assembly behavior as a function of the average concentration $\bar{c}$. Fig. 9, represents the concentration $c_{p}$ of each particle averaged in a time interval where no concentration switch is observed, as a function of the average concentration $\bar{c}$ of the assembly. The semi-grand canonical concentrations $c_{1}$ and $c_{2}$ correspond to the red dashed lines and when $\bar{c}$ fulfills Eq. 19, the concentration of each particle is highlighted in red. The dotted lines are guidelines. For $\bar{c} \leq c_{1}$ and for $\bar{c} \geq c_{2}$, each particle concentration is $\bar{c}$ (single-phase domain). For the five concentrations $\bar{c}$, which fulfill Eq. 19, the equilibrium concentrations calculated in the canonical (red symbols) are identical to those calculated in the semi-grand canonical ensemble (dashed lines). When $\bar{c}$ is outside the discrete spectrum, $c_{I I}$ is close to $c_{2}$, whereas $c_{I}$ adopt a quasi linear behavior (dotted lines): 


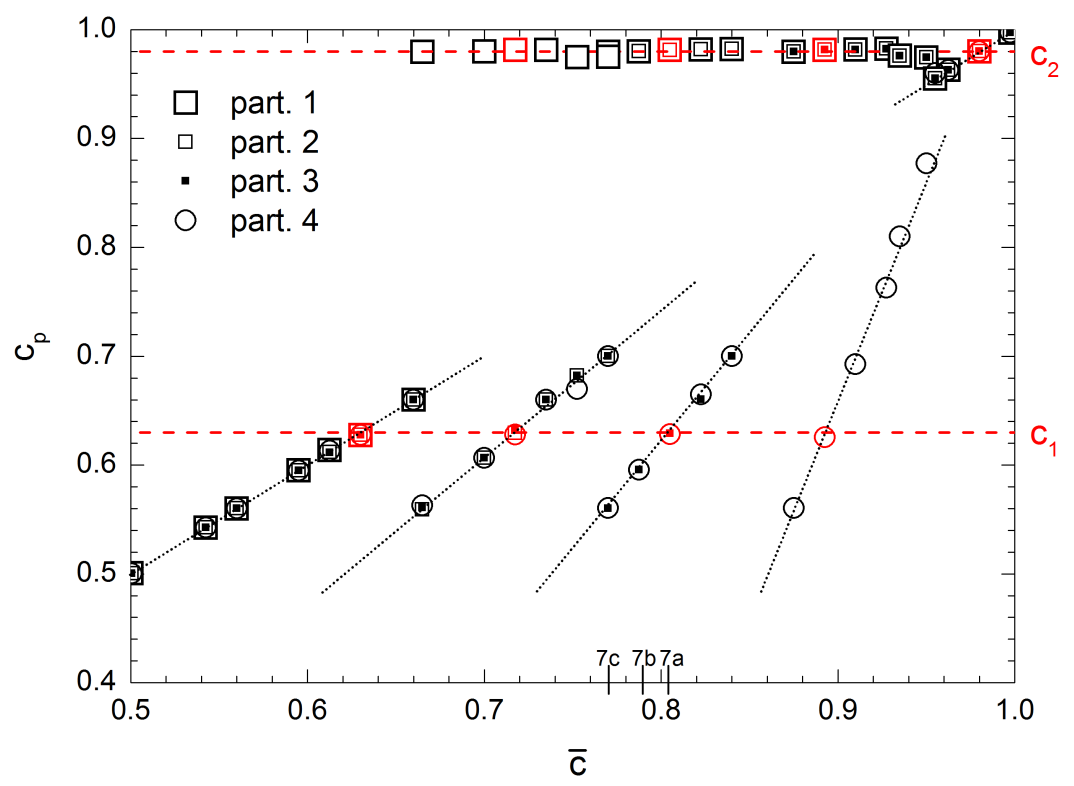

FIG. 9. Single particle concentration $c_{p}(\mathrm{p}=1,2,3,4)$ versus particle assembly concentration $\bar{c}$, obtained by Monte Carlo simulations in the canonical ensemble at 400K in a 4-particle system. The particle diameter is $2.2 \mathrm{~nm}$. Red dashed lines correspond to the equilibrium concentrations $\mathrm{c}_{1}=0.63$ and $c_{2}=0.98$ calculated in the semi-grand canonical ensemble. Red symbols are the measured concentrations when $\bar{c}$ fulfills Eq. 19 and dotted lines correspond to the extrapolation of Eq. 20. Labels $7 a, 7 b$ and $7 c$ denote the concentrations $\bar{c}$ analyzed in Fig. 7 .

$$
c_{I} \approx \frac{N \bar{c}-N_{I I} c_{I I}}{N_{I}}
$$

which is simply a consequence of the conservation law, given that $c_{I I}$ is hardly sensitive to $\bar{c}$. Finally, we note that for $\bar{c}=0.96$, the four particles adopt the same state. This means that in the canonical ensemble, the single state domain is reached before the semi-grand canonical limit $\mathrm{c}_{2}=0.98$, as it should.

\section{B. Finite assemblies of particles with different sizes}

The synthesis of nanoalloys by the physical route is often obtained by the deposition of atoms on a substrate. During the deposition, the substrate is usually heated to enhance 
diffusion and a post-synthesis heat treatment is sometimes added. At the end of such a process, the particles are in a coarsening regime with low thermodynamic driving forces. In binary nanoalloys, where the exchange of atoms between particles is much faster for a given species than for the other one, a partial equilibrium is reached leading to particle assemblies in which the particle concentration is size dependent. ${ }^{23}$

In the present work, the importance of the size distribution of a particle assembly is demonstrated through the computation of the canonical equilibrium of an assembly with a prescribed size distribution. We consider 30 particles assembly made of three sub-assemblies: 5 nanoparticles with a diameter of $2.2 \mathrm{~nm}, 15$ with a diameter of $4.4 \mathrm{~nm}$ and 10 with a diameter of $6.6 \mathrm{~nm}$. As in the previous section, the number of atomic sites of each particle is fixed and Monte Carlo simulations are performed to reach equilibrium in the canonical ensemble where the number for A and B atoms in the assembly, the temperature and pressure are fixed quantities.

Fig. 10 represents the equilibrium concentration $c_{p}$ of each particle at $T=400 \mathrm{~K}$ as a function of the nominal concentration $\bar{c}$ of the assembly in $\mathrm{B}$ atoms. For concentrations lower than $\bar{c}=0.45$, all the sub-assemblies are in a single-population state and it is observed that the smaller the particle size, the higher the concentration in B atoms. This effect is due to the segregation of $B$ atoms at the surface and to the fact that the relative number of surface sites is higher in smaller nanoparticles. For a concentrations $\bar{c}$ above 0.34, the smaller nanoparticles (2.2 nm in diameter), contain almost only B atoms. Therefore, an increase of the assembly concentration will only influence the $4.4 \mathrm{~nm}$ and $6.6 \mathrm{~nm}$ sub-assemblies.

For $0.46<\bar{c}<0.6$, the concentrations of the particles in the $4.4 \mathrm{~nm}$ and $6.6 \mathrm{~nm}$ subassemblies are independent of $\bar{c}$. All particles in the $6.6 \mathrm{~nm}$ sub-assembly have a concentration close to 0.3 . The $4.4 \mathrm{~nm}$ sub-assembly splits into two populations with compositions either close to $c_{I}=0.58$ or $c_{I I}=0.98$. In this composition range, an increase of the concentration $\bar{c}$ only implies a change of the number of particles between these two populations.

For a composition above $\bar{c}=0.6$, both the $2.2 \mathrm{~nm}$ and $4.4 \mathrm{~nm}$ sub-assemblies are almost saturated in $\mathrm{B}$ atoms. For $0.6<\bar{c}<0.72$, the $6.6 \mathrm{~nm}$ particle sub-assembly is in a singlepopulation state, and the particle concentration is linearly increasing with $\bar{c}$ as expected from the conservation of the number of $\mathrm{B}$ atoms. Finally, above $\bar{c}=0.75$, a two population domain takes place for the largest particles.

To highlight the consequences of a size distribution, we now summarize how the above 


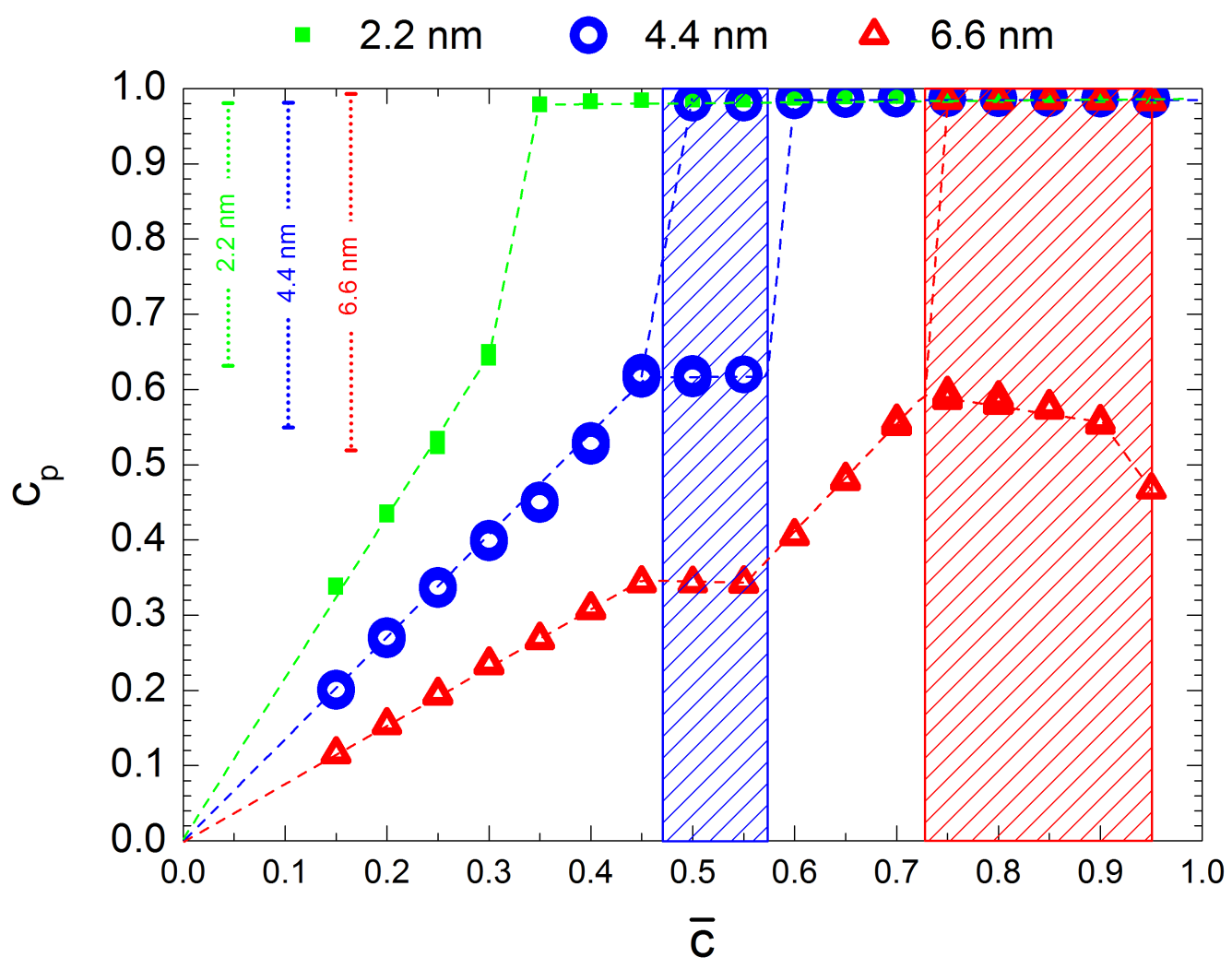

FIG. 10. Particle concentration $c_{p}$ as function of the assembly concentration $\bar{c}$ obtained with Monte Carlo calculations in the canonical ensemble, at $T=400 \mathrm{~K}$, for a 30 particle assembly with different sizes $(2.2 \mathrm{~nm}, 4.4 \mathrm{~nm}$ and $6.6 \mathrm{~nm}$, see text for detail). Dashed lines are guidelines and dotted lines correspond to the two-phase domains obtained in the semi-grand canonical calculations.

results compare with the behavior of infinite assemblies of monodisperse particles obtained in the semi-grand canonical calculations (see Sec. III B). First, we found in both cases that the equilibrium particle concentration is size dependent. Second, particles of a given size forming a sub-assembly may all have the same composition or be split into two populations with different equilibrium compositions $c_{I}$ and $c_{I I}$. The latter compositions are close to the semigrand canonical equilibrium compositions $c_{1}$ and $c_{2}$. We also found that the concentration ranges where each sub-assembly is in a two-population state can significantly differ from the semi-grand canonical prediction. This results from the difference between the equilibrium composition of each sub-assembly and the average composition. This difference is expected to be large in the present work where surface segregation occurs because the fraction of 


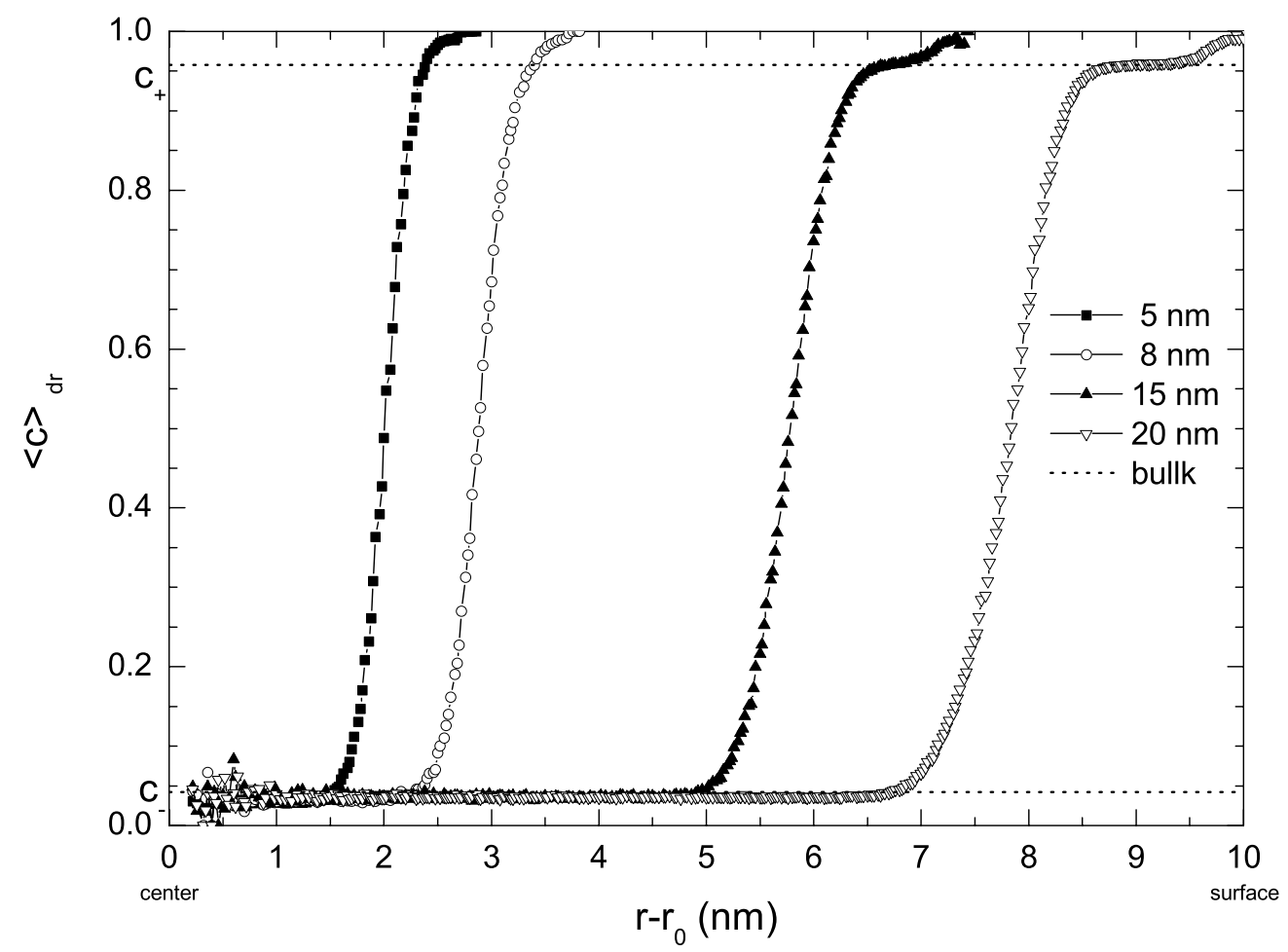

FIG. 11. Averaged radial concentration profiles of $5 \mathrm{~nm}, 8 \mathrm{~nm}, 15 \mathrm{~nm}$ and $20 \mathrm{~nm}$ isolated particles with $\bar{c}=0.5$ at $500 \mathrm{~K} . \mathrm{c}_{+}$and $\mathrm{c}_{-}$correspond to the solubility limits of the bulk alloy (dotted lines).

surface sites is directly related to the particle size.

\section{THERMODYNAMICS OF ISOLATED PARTICLES}

We have shown in the previous section that, when assemblies of particles with identical size are considered, the canonical equilibrium can be characterized by a coexistence of two particle populations which differ by their averaged concentration, a behavior formally similar to the phase separation observed in bulk alloys. In this part, we consider the stability of an isolated particle, and we question whether a phase separation can be observed within the particle. To allow a meaningful comparison with the bulk case, and with the results obtained on particle assemblies (Sect. III B), the same binary Lennard-Jones system is used (Tab. I) for which A and B atoms have the same size and a segregation of B atoms at the surface is 


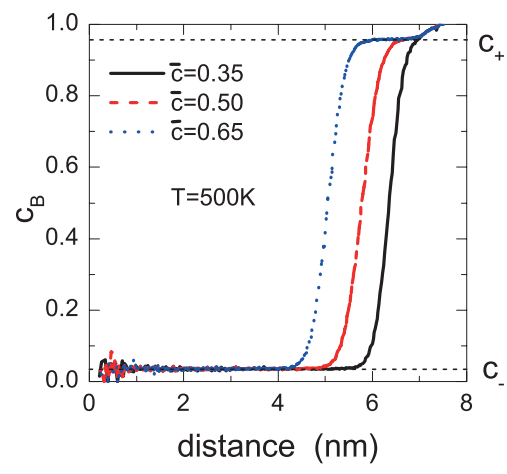

(a)

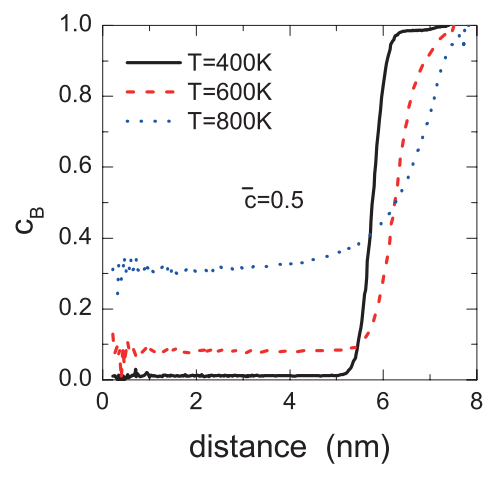

(b)

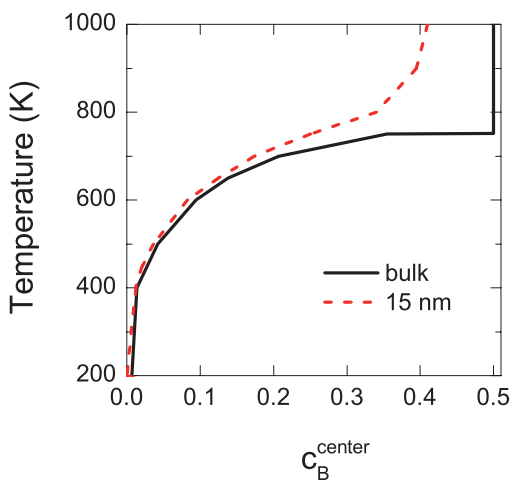

(c)

FIG. 12. Concentration (a) and temperature (b) dependence of the averaged radial concentration profiles in a particle with a diameter equal to $15 \mathrm{~nm} . \mathrm{c}_{+}$and $\mathrm{c}_{-}$correspond to the solubility limits of the bulk alloy (dotted lines). (c) is a comparison between the concentration measured in the center of the particle (dashed line) and the solubility limit of the bulk alloy (solid line) for $\bar{c}=0.5$.

expected. We only consider in this section large enough nanoparticles so that a core region and a surface region can be distinguished. Our aim is to analyze whether a two-phase state can be properly defined in the particle and how phase diagram can be built in this context.

We consider spherical particles of a given radius in the canonical ensemble where the number of $\mathrm{A}$ and $\mathrm{B}$ atoms, the temperature and the pressure are fixed quantities. For the concentrations investigated here, the concentration fields observed in the particle have a radial symmetry and the equilibrium can be analyzed in terms of radial concentration profiles.

Equilibrium concentration profiles for different particle diameters $D$ are presented in Fig. 11. The same average concentration $\bar{c}=0.5$ is used and the temperature $T=500 \mathrm{~K}$ is chosen well below the bulk critical temperature $\left(T_{c}^{b u l k}=750 \mathrm{~K}\right)$. As expected, the larger particles $(D \geq 15 \mathrm{~nm})$ display two homogeneous regions whose concentrations are close to the bulk solubility limits (dashed lines) and the volume fraction of these regions is close to the value 0.5 predicted by the lever rule. In addition, a segregation of B atoms is observed at the particle surface. The concentration profile inside smaller particles $(5 \mathrm{~nm} \leq D \leq 8 \mathrm{~nm})$ is qualitatively different. Indeed, a similar low concentration phase is observed in the core of the particle $\left(\mathrm{c}=\mathrm{c}_{-}\right)$, but the concentration profile then continuously increases to reach a value close to 1 at the surface. This means that for small particles, the high concentration 
phase (expected in the bulk case) does not appear due to the segregation of B atoms at the surface. In brief, at $\bar{c}=0.5$, and at a temperature $250 \mathrm{~K}$ below the bulk critical temperature, the equilibrium state of a particle can only be described as a mixture of two homogeneous phases (with concentrations close the bulk solubility limits) if the particle diameter is above $8 \mathrm{~nm}$.

In Fig. 12, we analyze in more detail the equilibrium state of a rather large nanoparticle (diameter $\mathrm{D}=15 \mathrm{~nm}$ ). For this particle size, the coexistence at equilibrium of two homogeneous phases is investigated when changing the average particle concentration (Fig. 12a) and the temperature (Fig. 12b). When the shell of the particle contains an homogeneous phase, a plateau (close to $\mathrm{c}_{+}$) is observed in the radial concentration profile. More precisely, we have considered that a homogeneous shell phase was detected as soon as an inflection point could be measured in the concentration profile. Fig. 12a shows that at $T=500 \mathrm{~K}$, the equilibrium microstructure of a nanoparticle with an average concentration above 0.4 is made of two homogeneous phases with concentrations that are close to the bulk solubility limits $\left(\mathrm{c}_{-}, \mathrm{c}_{+}\right)$. This is no more the case for concentrations below 0.4 and the concentration profile is continuously increasing between the value of the core phase and 1 at the surface. This behavior is similar to the one presented in Fig. 11 for small particles and originates from the strong segregation of B atoms at the surface.

Fig. 12b shows that the concentration profile inside of nanoparticle with an average concentration $\bar{c}=0.5$ qualitatively changes with temperature. The microstructure containing two homogeneous phases is stable at low temperature, then a transition towards the microstructure composed of only one homogeneous phase at the core is observed at $T=600 \mathrm{~K}$. Note finally that for the temperature above the bulk critical temperature, the concentration profile is not constant because of the strong segregation of B atoms at the surface. In that situation, a rather homogeneous core concentration can still be defined even if its value is far from the average composition expected in the bulk case.

The evolution of the concentration of the core of the particle as a function of the temperature is presented in Fig. 12c (red dashed line) for $\bar{c}=0.5$. Below the bulk critical temperature $T_{c}^{b u l k}$, the core concentration is close to the solubility limit. Above $T_{c}^{b u l k}$, the core concentration continuously increases to concentrations closer to the average concentration $\bar{c}=0.5$. An important point is that the transition between these two regimes is continuous. The discontinuity observed in bulk systems at the transition can not be observed in nanoparticles 
because of their finite size character.

The results presented in Fig. 12 show that, even for rather large nanoparticles $(D=$ $15 \mathrm{~nm}$ ), the equilibrium configuration is quite complex but can be approximately described in terms of one or two homogeneous phases supplemented by an heterogeneous layer of B atoms at the surface. Therefore, for large enough particle sizes, a composition-temperature phase diagram can be constructed to defined domains in which two homogeneous phases are simultaneously observed at equilibrium. This phase diagram is presented in Fig. 13 (red dashed line). It is clear that in a nanoparticle of $15 \mathrm{~nm}$ in diameter, the two-phase stability region is much smaller than in the bulk case. This large difference is due to the size effect of the nanoparticle combined with a rather strong segregation of B atoms at the surface. Indeed, all the B atoms that have segregated at the surface are no more available to form a two-phase state within the particle. Note however that contrary to bulk alloys, the limits of the two-phase domain do not correspond to the phase concentrations observed within the particle. Therefore, additional graphs such as the one presented in Fig. 12c are necessary to fully describe the internal state of the particle. As an example, when comparing Fig. 12c and Fig. 13, it appears clearly that, even if the particle size effect strongly modifies the two-phase domain limits, the core concentration which is observed when the concentration profile displays a two-phase structure deviates only slightly from the low concentration limit of the bulk phase diagram. A complete description would also require a detail description of the segregation process which is beyond the scope of the present paper (see for example Refs. 19 and 1).

Finally, we recall that we have selected in the present analysis rather large particles to analyze how the usuall description by a phase diagram has to be modified and extended to describe the equilibrium state of a binary nanoalloy. When considering small nanoparticles a description based on a coexistence of homogeneous phases (eventually supplemented by a segregation layer) can be irrelevant. As an example, the concentration profiles corresponding to a nanoparticle of $6 \mathrm{~nm}$ in diameter are presented in Fig. 14. In that case, the concentration profile continuously increases from the center to the outer shell so that no homogeneous region can be defined within the particle when the temperature is above $600 \mathrm{~K}$. The proper description of such situations requires an atomistic approach, in which the atomic sites of the particle are automatically treated as nonequivalent. Intermediate approaches could also be of interest, where sites are divided in several sets (surface atoms, first sub-surface atoms, 


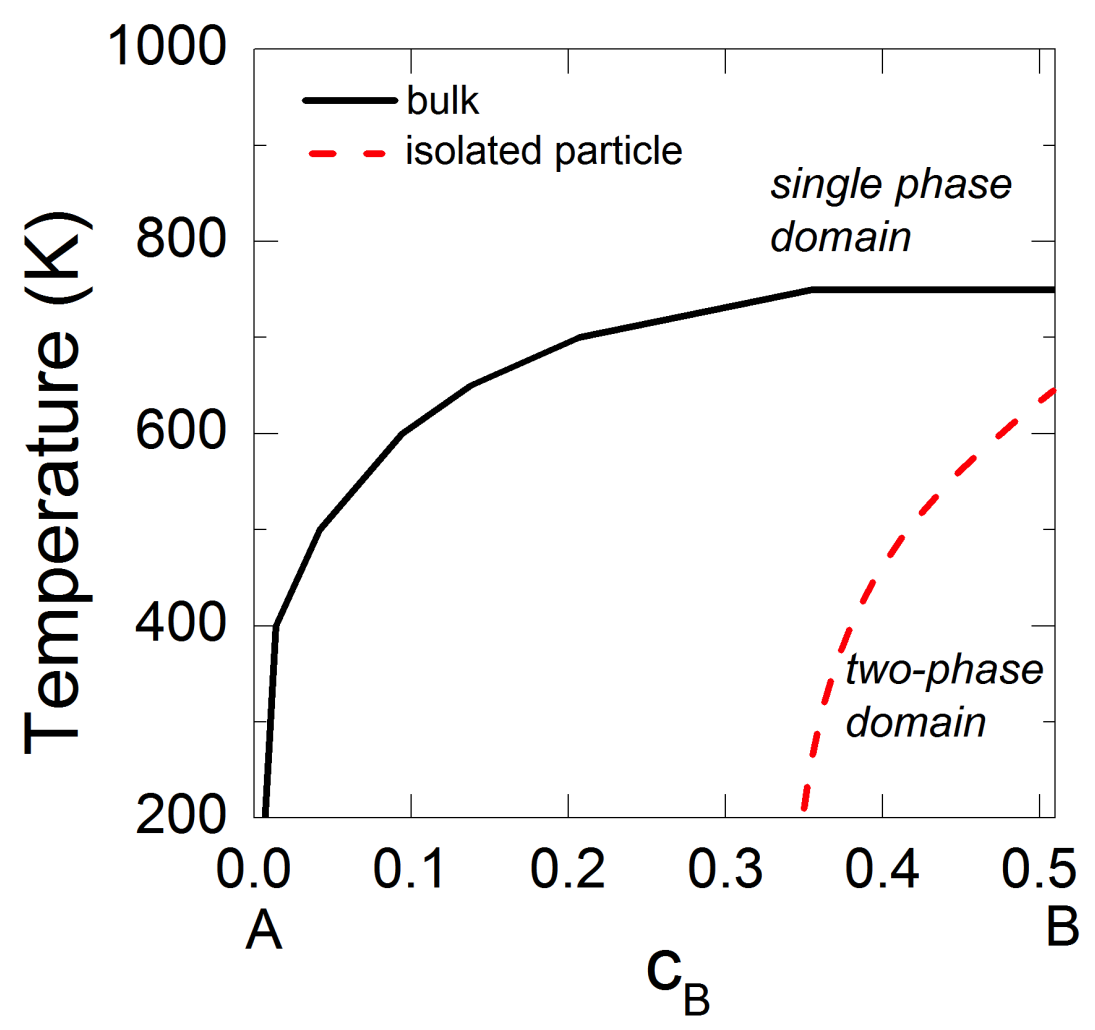

FIG. 13. Composition-temperature phase diagram for a bulk alloy and for an isolated particle with a diameter equal to $15 \mathrm{~nm}$ (see text for detail).

...), in which all sites are assumed equivalent. ${ }^{19}$

\section{CONCLUSION}

In this work, we studied the thermodynamics of binary fcc nanoparticles using a simple atomic pair potential. Equilibrium states were computed with off-lattice Monte Carlo simulations in several thermodynamic ensembles.

In the semi-grand canonical ensemble, the $(\Delta \mu, T)$ phase diagram and the resulting $(c, T)$ phase diagram were computed for particle size between $2.2 \mathrm{~nm}$ and $13 \mathrm{~nm}$ and compared to the bulk case. When decreasing the particle size, we obtained a significant decrease of the solid/liquid transition temperatures as well as a growing asymmetry of the solid state miscibility gap related to surface segregation effects. The $(c, T)$ phase diagram obtained in the semi-grand canonical ensemble describes the stability of an infinite assembly of particles 


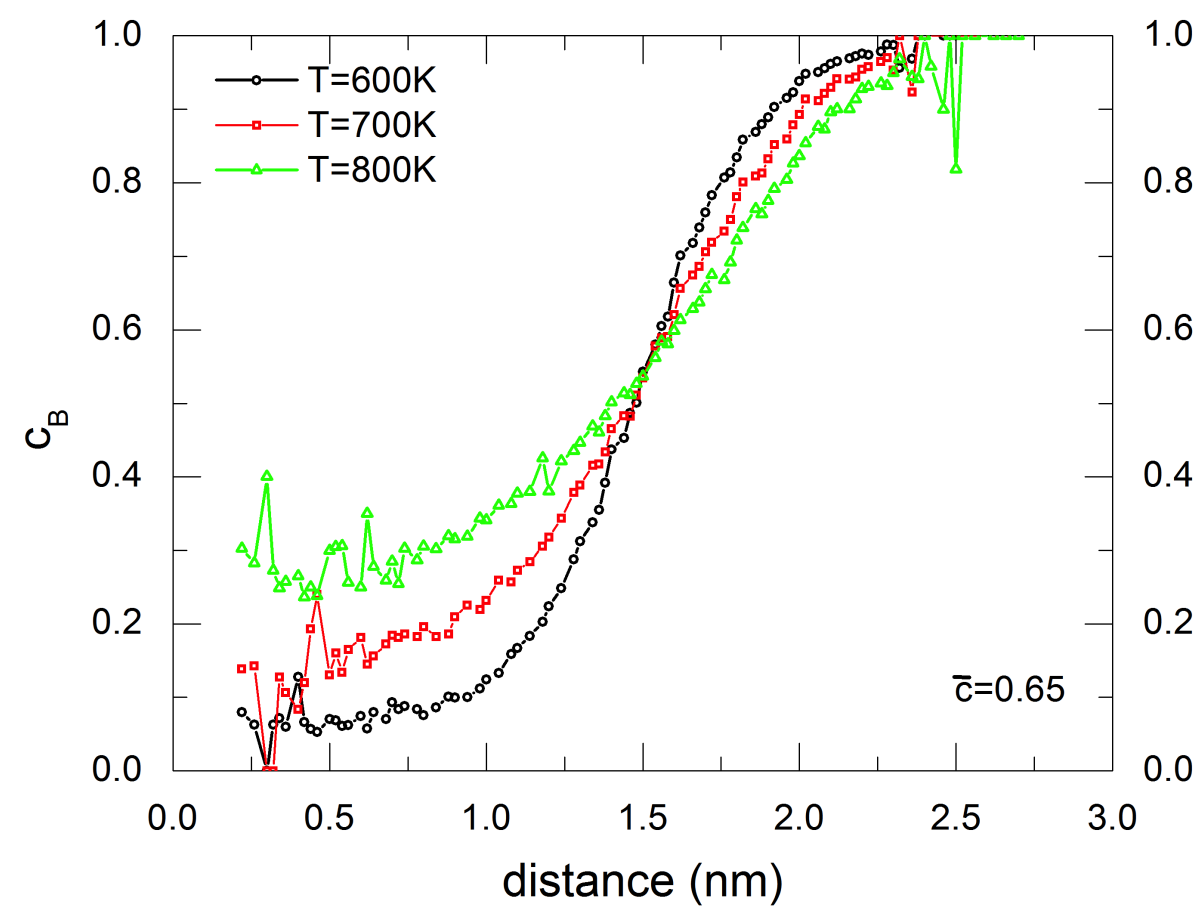

FIG. 14. Concentration profiles calculated at different temperatures in a particle with a diameter equal to $6 \mathrm{~nm}$ and an average concentration equal to 0.65 .

of the same size:

- within the one-phase stability regions, all particles are identical at equilibrium

- within the two-phase stability regions the assembly splits in two subassemblies with average concentrations equal to the solubility limits.

Note that this phase diagram gives no information on the repartition of concentration inside each particle. Additional diagrams are needed to describe this information.

We then studied the stability of finite assemblies of particles using a canonical ensemble in which particles only interact by the exchange of atoms. When considering particles of identical sizes, we showed, using a general thermodynamic formulation of the problem, that the equilibrium configuration is either an assembly of identical particles or a set of two such assemblies. In the latter case, the concentrations of the two sub-assemblies are equal to the semi-grand canonical equilibrium compositions only for certain quantified values of the 
average concentration. These predictions were confirmed by simulations of different finite monodisperse assemblies of particles.

We then modeled the importance of a size distribution by computing the canonical equilibrium of an assembly composed of three sub-assemblies with three different sizes. First, a strong size effect was evidenced; small particles tends to be enriched in the segregating $\mathrm{B}$ atom, because of their larger surface to volume ratio. Second, we found that particles belonging to a given sub-assembly can either all adopt the same composition or be split in two populations. As a conclusion, the equilibrium of the particle assembly with a size distribution combines a size effect and the fact that a given particle size may adopt two configurations.

Finally, we have considered the thermodynamics of an isolated particle to analyze whether a phase separation can be defined within a particle. When studying rather large nanoparticles (15 nm in diameter) we showed that the equilibrium state can still be described by either a homogeneous phase or a two-phase configuration, supplemented by a segregation layer. We found that the region in which a two-phase domain can be identified inside a particle is well below the bulk phase diagram. However, in this region, the concentration of the homogeneous core remains very close to the bulk solubility limit. We also showed that the top-down description of the stability of nanoparticles based on homogeneous phases supplemented by surface properties may be irrelevant for small nanoparticles, especially at high temperature.

The semi grand canonical (SGC) ensemble was essentially used to rationalize the results obtained within the canonical ensembles of mono-disperse particles. Notwithstanding this point, we did consider canonical assemblies of poly-disperse particles. This procedure enabled the analysis of the equilibrium state of particles with prescribed sizes, even though a complete analysis of such assemblies should of course be done within a context of out-ofequilibrium thermodynamics. Also, a natural extension of this work is a systematic study of the consequences of an atomic size mismatch on the equilibrium configuration of nanoparticle. Because the elastic driving force is usually very anisotropic, a strong impact on the configuration of particles is expected..$^{25-27}$ In addition, such an off-lattice method could be used to clarify the formation of misfit dislocations at the nanoparticle interface. ${ }^{6,28}$ 


\section{ACKNOWLEDGMENTS}

The authors acknowledge the networking support of the COST Action MP0903 NANOALLOY.

* Mathieu.Fevre@onera.fr

1 D. Alloyeau, C. Mottet, and C. Ricolleau Nanoalloys. Synthesis, Structure and Properties (Springer-Verlag, London, 2012).

2 W. A. Jesser, R. Z. Shneck, and W. W. Gile, Phys. Rev. B 69, 144121 (2004).

3 D. Alloyeau, C. Ricolleau, C. Mottet, T. Oikawa, C. Langlois, Y. Le Bouar, N. Braidy, and A. Loiseau, Nat. Mater. 8, 940 (2009).

4 K. Sato, A. Kovács, and Y. Hirotsu, Thin Solid Films 519, 3305 (2011).

5 A.S. Shirinyan, A.M. Gusak, and M. Wautelet, Acta Mater. 53, 5025 (2005).

6 N. Braidy, G.R. Purdy, and G.A. Botton, Acta Mater. 56, 5972 (2008).

7 A.M. Gusak, A.O. Kovalchuk, and B.B. Straumal, Philos. Mag. 93, 1677 (2013).

8 Y. Le Bouar, in Mechanics of nano-objects, Les Presses de l'École des Mines de Paris, 213 (2011).

9 M.H. Ghatee, and K. Shekoohi, Fluid Phase Equilib. 355, 114 (2013).

10 L. Delfour, J. Creuze, and B. Legrand, Phys. Rev. Lett. 103, 205701 (2009).

11 V. Moreno, J. Creuze, F. Berthier, C. Mottet, G. Tréglia, and B. Legrand, Surf. Sci. 600, 5011 (2006).

12 I. Atanasov, and M. Hou, Surf. Sci. 603, 2639 (2009).

13 F. Calvo, Phys. Chem. Chem. Phys. 17, 27922 (2015).

14 F. Ducastelle, in Nanoalloys. Synthesis, Structure and Properties (Springer-Verlag, London, 197 2012).

15 I. Atanasov, R. Ferrando, and R. L. Johnston, J. Phys.: Condens. Matter 26, 275301 (2014).

16 F. Baletto, and R. Ferrando, Rev. Mod. Phys. 77, 371 (2005).

17 N. Metropolis, A. W. Rosenbluth, M. N. Rosenbluth, A. H. Teller and E. Teller, J. Chem. Phys. 21, 1087 (1953).

18 M. Fèvre, C. Varvenne, Y. Le Bouar, and A. Finel, Philos. Mag. 93, 1563 (2013). 
19 F. Lequien, J. Creuze, F. Berthier, I. Braems, and B. Legrand, Phys. Rev. B 78, 075414 (2008).

20 R. Tétot, F. Berthier, J. Creuze, I. Meunier, G. Tréglia, and B. Legrand, Phys. Rev. Lett. 91, $176103(2003)$.

21 M. Polak, and L. Rubinovich, Surf. Sci. 584, 41 (2005).

22 H. Haberland, T. Hippler, J. Donges, O. Kostko, M. Schmidt, and B. von Issendorff, Phys. Rev. Lett. 94, 035701 (2005).

23 G. Prévot, N.T. Nguyen, D. Alloyeau, C. Ricolleau, and J. Nelayah, ACS Nano 10, 4127 (2016).

24 F. Calvo, and C. Mottet, Phys. Rev B 84, 035409 (2011).

25 C. Langlois, D. Alloyeau, Y. Le Bouar, A. Loiseau, T. Oikawa, C. Mottet, and C. Ricolleau, Faraday Discuss. 138, 375 (2008).

26 R. Ferrando, J. Phys. Condens. Matter. 27, 013003 (2015).

27 J.-P. Palomares-Baez, E. Panizon, and R. Ferrando, Nano Lett. 17, 5394 (2017).

28 N. Bhattarai, G. Casillas, A. Ponce, and M. Jose-Yacaman, Surf. Sci. 609, 161 (2013). 